\title{
Random Surface Correlation Functions
}

\author{
D. B. Abraham ${ }^{1, \star}$, J. T. Chayes ${ }^{2, \star \star}$, and L. Chayes ${ }^{2, \star \star}$
}

1 Department of Mathematics, University of Melbourne, Parkville, Vic. 3052, Australia

2 Department of Physics, Harvard University, Cambridge, MA 02138, USA

\begin{abstract}
Truncated pair functions for free random surface models and Bernoulli ensembles are examined. In both cases, the pair function is shown to obey Ornstein-Zernike scaling whenever various correlation lengths of the system satisfy a nonperturbative criterion. Under the same conditions, the transverse displacement of surfaces contributing to the pair function is shown to be normally distributed. A new type of transition, which concerns the width of typical surfaces, is introduced and studied. Whenever the system is below the melting transition temperature of a related lower-dimensional model, the width of typical surfaces is shown to be finite. A thermodynamic formalism for free random surface models is developed. The formalism is used to obtain sharp estimates of the entropy of surfaces contributing to the pair function.
\end{abstract}

\section{Introduction}

The stochastic geometry of random surfaces has recently become a topic of considerable interest (see [1] and references therein). The correlation functions of lattice gauge theories, three-dimensional spin systems and models of crystalline interfaces have natural expressions as weighted sums over surfaces. However, such expressions are difficult to analyze due to both the combinatoric problems introduced by the large number of surfaces, and the intractability of explicit forms for the associated weights. It is therefore of interest to study models of correlation functions which are defined as sums over restricted classes of surfaces with relatively simple weights.

In this paper, we analyze the beahvior of correlation functions of the form

$$
Q_{\mathscr{S}}(\beta)=\sum_{S \in \mathscr{S}} e^{-\beta|S|},
$$

* On leave from Department of Theoretical Chemistry, Oxford University, Oxford OX1 3TG, England

$\star \star$ Work partially supported by the National Science Foundation under Grant No. PHY8203669 
where $\beta \sim g_{0}^{-2}$ is the inverse temperature or the square of the inverse coupling, $\mathscr{S}$ denotes some preprescribed class of surfaces on the lattice $\mathbb{Z}^{d}$, and $|S|$ is the area (i.e., the number of plaquettes) of the surface $S \in \mathscr{S}$. We also study the analogue of $Q_{\mathscr{S}}$ for a system of Bernoulli plaquettes. In this case, the weights in (1.1) are replaced by the probabilities of (disjoint) events which correspond to the surfaces in $\mathscr{S}$.

Our principal results, some of which were announced in [2], concern truncated pair functions (i.e., glue-ball propagators), $Q_{\mathscr{S}_{L}}(\beta) \equiv Q_{L}(\beta)$. Here $\mathscr{S}=\mathscr{S}_{L}$ denotes some set of tubular surfaces which have as their boundary two plaquettes separated by a distance of $L$ lattice units along a particular axis. These surfaces may be generated by the time evolution of a ring obeying certain constraints. The result is a class of self-avoiding surfaces with a solid-on-solid (SOS) character in the "time" direction.

The quantity $Q_{L}(\beta)$ serves as an approximation to the low temperature expansion of the truncated pair correlation in a three-dimensional ferromagnet. [By duality, $Q_{L}(\beta)$ also models the strong coupling expansion of the corresponding, Ising-type gauge theory.] Such random surface approximations are expected to be quite accurate in the low temperature regime. Indeed, the analogous twodimensional path expansion has been solved exactly [3]. It quantitatively reproduces all known results for the Ising pair correlation in $d=2$.

Random surface phenomena in a system of Bernoulli plaquettes have been studied in [4]. Our analogue of $Q_{L}(\beta)$ for such a system is defined in terms of the probability that a configuration containing a surface in $\mathscr{S}_{L}$ occurs. We expect the quantity so defined to approximate the actual truncated pair correlation for the entire range of the parameter in the Bernoulli system.

Analogues of $Q_{L}(\beta)$ may be defined for classes of surfaces other than $\mathscr{S}_{L}$. For example, if the sum is performed over planar surfaces, the resulting function is expected to model the propagator of $\mathrm{SU}(N)$ theories in the large $N$ limit. Such models have been studied in detail by Durhuus, Fröhlich and Jónsson $[5,6]$.

Our first results, established in Sect. 2, concern the long-distance scaling of $Q_{L}(\beta)$. We show that, for $\beta$ sufficiently large,

$$
Q_{L}(\beta) \sim \frac{1}{L^{(d-1) / 2}} e^{-M(\beta) L} \quad \text { as } \quad L \rightarrow \infty,
$$

where $M(\beta)$ is the glue-ball mass or inverse correlation length. Two ingredients are necessary for the proof of (1.2). First we demonstrate that $M(\beta)$ exists and is strictly positive above some melting point $\beta_{c}$. We then develop a random surface OrnsteinZernike equation which enables us to establish the power law corrections indicated in (1.2). We also show that the Ornstein-Zernike equation allows us to obtain monotone approximants to the mass $M(\beta)$.

In Sect. 3, we define a truncated pair function for an ensemble of Bernoulli plaquettes at density $p$. It is shown that this pair function obeys the scaling (1.2) for $p$ sufficiently small.

The properties of typical surfaces which contribute to the pair functions are investigated in Sect. 4. We first note that the motion of tubes is normally distributed, so that the typical tube wanders as $\sqrt{L}$. Next, we consider fluctuations 
in the width of typical tubes. It is shown that whenever $\beta$ is larger ( $p$ is smaller) than the melting transition point for a related lower-dimensional model, the expected width of the tube is finite.

For analysis of the entropy of random surfaces, it is convenient to write

$$
Q_{L}(\beta)=\sum_{k} \Gamma_{k}(L) e^{-\beta k L},
$$

where $\Gamma_{k}(L)$ is the number of surfaces with exactly $k L$ plaquettes. Thus $k$ may be viewed as a covering factor.

In Sect. 5, we establish that the number of tubes grows exponentially in $L$ for fixed $k$. Moreover, for $k$ sufficiently close to its minimum value, we determine the power law corrections to exponential growth:

$$
\Gamma_{L}(k) \sim \frac{1}{L^{d / 2}} e^{\zeta(k) L} \quad \text { as } \quad L \rightarrow \infty .
$$

The significance of the covering factor $k$ and the corresponding entropy $\zeta(k)$ for (general) random surface models of the form (1.1) is discussed in some detail. It is shown that the covering factor is canonically conjugate to the inverse temperature. Moreover, the mass $M(\beta)$ (or its analogue for other random surface correlations) is related to the entropy $\zeta(k)$ by a Legendre transform. This enables us to characterize critical properties of the model in terms of the behavior of $\zeta(k)$. In particular, we use estimates on $\zeta(k)$ to show that our pair function $Q_{L}(\beta)$ undergoes a catastrophic "phase transition" at some $\bar{\beta}>0$, in such a way that the model ceases to exist for $\beta<\bar{\beta}$. Moreover, we establish that the mass $M(\beta)$ approaches its limiting value $M(\bar{\beta})$ continuously as $\beta \downarrow \bar{\beta}$.

Finally, we show that the mass $M(\beta)$ is analytic for $\beta$ sufficiently large. The power law corrections indicated in (1.4) are then obtained by exploiting the relationship between the mass and the entropy.

Many of our results follow, either directly or indirectly, from the random surface Ornstein-Zernike equation established in Sect. 2. It is worth noting that such an equation is nonperturbative. Thus the region of validity of our results, alluded to earlier (i.e., " $\beta$ sufficiently large," " $p$ sufficiently small;" " $k$ sufficiently close to its minimum value"), is characterized by a nonperturbative criterion involving the relative size of various correlation lengths in the system.

\section{Asymptotic Decay of SOS Pair Correlations}

In this section we study a truncated pair correlation of the form (1.1) for the classes of surfaces $\mathscr{S}_{L}$ and $\mathscr{S}_{L}^{\prime}$ defined below. The principal result of this section is a proof of Ornstein-Zernike scaling [cf. Eq. (1.2)] of the pair function.

For clarity of exposition, throughout our analysis we restrict attention to the three-dimensional cubic lattice $\mathbb{Z}^{3}$. (See, however, the remark following the proof of Theorem 2.7.) The surfaces in $\mathscr{S}_{L}$ and $\mathscr{S}_{L}^{\prime}$ will be constructed from plaquettes on the dual lattice. We denote by $p_{0}$ the edges of the plaquette centered at $(1 / 2,0,0)$, and by $p_{L}$ the translation of $p_{0}$ through $L$ units in the $x$-direction. The plane $x=k$ will be denoted by $\mathbb{P}_{k}$. 
Definition. $\mathscr{S}_{L}^{\prime}$ is the set of all connected, self-avoiding surfaces $S$ with boundary $\partial S$ $=p_{0} \cup p_{L}$, satisfying the condition that the intersection of $S$ with each of the planes $\mathbb{P}_{k}, 1 \leqq k \leqq L$, is a single, closed ring $r_{k} \equiv S \cap \mathbb{P}_{k}$ (see Fig. 1).

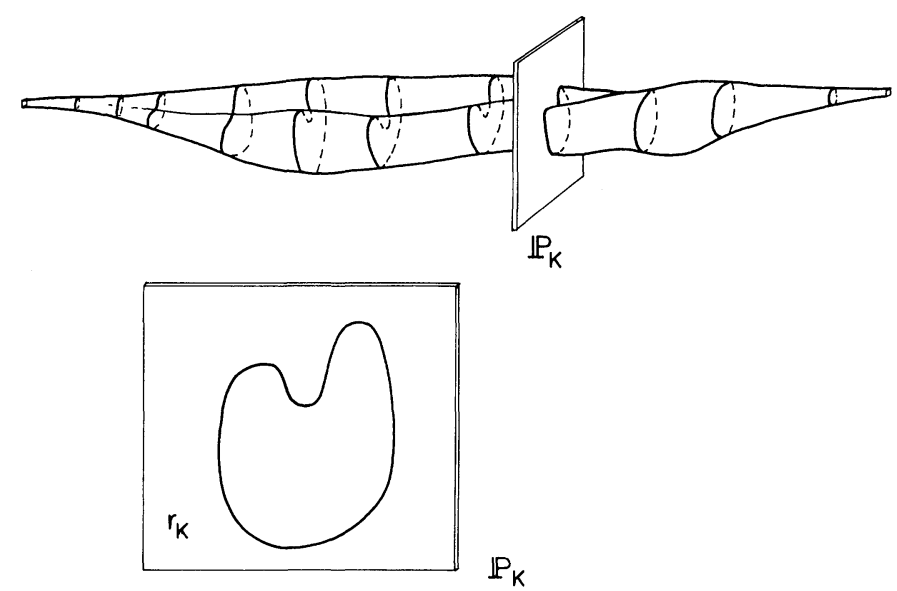

Fig. 1. A tube in $\mathscr{S}_{L}^{\prime}$

For convenience, we will actually study the subclass $\mathscr{S}_{L} \subset \mathscr{S}_{L}^{\prime}$ of surfaces for which the rings $r_{1}$ and $r_{L}$ are required to be elementary squares. In other words, the tubular surfaces in $\mathscr{S}_{L}$ begin and end on open boxes of four plaquettes surrounding the points $(1,0,0)$ and $(L, 0,0)$, respectively. Such boxes will be called "elementary chimneys." The restriction to $\mathscr{S}_{L}$ causes no loss of generality, since the pair correlations $Q_{L}=\sum_{S \in \mathscr{S}_{L}} e^{-\beta|S|}$ and $Q_{L}^{\prime}$ are related by

$$
Q_{L+2}=e^{-8 \beta} Q_{L}^{\prime} .
$$

We shall also be concerned with surfaces in ${ }_{L} \subset \mathscr{T}_{L}$ which satisfy the additional restriction that each of the rings $r_{k}$ surround the origin of $\mathbb{P}_{k}$. For obvious reasons, this subclass will be called the set of constrained tubes. The associated constrained pair correlation will be denoted by $q_{L}$.

Observe that the definition of $\mathscr{S}_{L}^{\prime}$ restricts us to surfaces which have no overhangs in the $x$-direction. For this reason, we shall call these surfaces solid-onsolid (SOS) tubes. However, it should be noted that our SOS tubes may have overhangs in the $y z$-planes.

We now establish some elementary results on the asymptotic behavior of $Q_{L}(\beta)$ for $L$ large.

Proposition 2.1. For all $\beta$,

$$
\lim _{L \rightarrow \infty} \log Q_{L}(\beta) / L \equiv-M(\beta)
$$

exists (in $\mathbb{R}^{*}$ ).

Proof. This follows from subadditivity. Indeed, those surfaces composed of a tube in $\mathscr{S}_{L_{1}}$ joined to a tube in $\mathscr{S}_{L_{2}}$ (which has been translated $L_{1}$ units in the $x$-direction) 
form a subset of $\mathscr{S}_{L_{1}+L_{2}}$. Thus

$$
Q_{L_{1}+L_{2}} \geqq Q_{L_{1}} Q_{L_{2}} .
$$

Corollary. $-M(\beta)$ provides a uniform upper bound on $\log Q_{L} / L$, i.e.

$$
Q_{L}(\beta) \leqq e^{-M(\beta) L} \quad \forall L .
$$

In particular, since $Q_{L} \geqq e^{-4 \beta L}$,

$$
M(\beta) \leqq 4 \beta \text {. }
$$

It can be shown, using standard methods, that the upper bound on $M(\beta)$ is exact as $\beta \rightarrow \infty$. In subsection (iii) we will outline a simple nonperturbative method by which one can obtain monotone approximants to the mass $M(\beta)$, thereby improving the bound (2.5).

Proposition 2.2. The mass $M(\beta)$ is a concave, nondecreasing function of $\beta$. Furthermore, $\exists 0<\beta_{c}<\infty$ such that $M(\beta)>0 \forall \beta>\beta_{c}$ and $M(\beta)<0 \forall \beta<\beta_{c}$.

Proof. That $M(\beta)$ is nondecreasing is obvious. To establish concavity, let $\lambda \in[0,1]$ and observe that $\forall L$

$$
Q_{L}\left(\lambda \beta_{1}+(1-\lambda) \beta_{2}\right) \leqq Q_{L}^{\lambda}\left(\beta_{1}\right) Q_{L}^{1-\lambda}\left(\beta_{2}\right)
$$

by the Hölder inequality.

To demonstrate the existence of $\beta_{c}$, observe that by a simple Peierls argument, if $\beta>\log 11$

$$
M(\beta) \geqq 4(\beta-\log 11)>0 .
$$

On the other hand, using (2.4) for $L=3$, we have

$$
e^{-3 M(\beta)} \geqq Q_{3}(\beta) \geqq e^{-12 \beta}+4 e^{-16 \beta},
$$

where the second inequality is obtained by considering only the five simplest surfaces in $\mathscr{S}_{3}$. The lower bound exceeds 1 for $\beta$ sufficiently small.

Remark. The concavity of $M(\beta)$ implies that it can have at most a single jump discontinuity. Should this occur at some $\bar{\beta}$, then $M(\beta)=-\infty$ for all $\beta<\bar{\beta}$. This means, of course, that the model does not exist for $\beta<\bar{\beta}$. In Sect. 5, we will establish that such a catastrophic "phase transition" does indeed occur at some $\bar{\beta}>0$. Moreover, we will show that $M(\bar{\beta})=\lim _{\beta \downarrow \bar{\beta}} M(\beta)$ - a result which is not implied by concavity alone. It is probable that $\bar{\beta}=\beta_{c}$; however, we do not yet have a proof.

We note that Propositions 2.1 and 2.2 are basically a consequence of the form of the pair correlation (1.1), and are not sensitive to the class of surfaces under consideration, provided they are subadditive. In particular the results obviously hold for constrained SOS tubes.

(i) Pure Exponential Decay for Constrained Tubes. We will now establish that the pair correlation $q_{L}(\beta)$ for constrained tubes decays via a pure exponential, i.e. for $\beta$ sufficiently large there exist constants $\kappa_{1}(\beta), \kappa_{2}(\beta)>0$ such that

$$
\left|q_{L}(\beta) e^{+m(\beta) L}-\kappa_{1}(\beta)\right| \leqq e^{-\kappa_{2}(\beta) L} .
$$


In the above expression, $m(\beta)$ denotes the mass for the constrained tubes [cf. Eq. (2.2)]. The quantity $\kappa_{2}(\beta)$ corresponds to the "upper gap" in the particle spectrum of the associated field theory. ${ }^{1}$

We observe that pure exponential decay of $q_{L}$ is consistent with the scaling in Eq. (1.2), since the constraint of encircling the $x$-axis forces the tubes in $\supset_{L}$ to be effectively one-dimensional.

In order to prove the result, we introduce a notion which is well known in theories of continuum fluids [7]: the direct correlation function. Recall that the surfaces in $\Im_{L}$ must begin (and end) on an elementary chimney. We may classify the surfaces in $\sigma_{L}$ according to the location of their next elementary chimney, i.e. the earliest point at which they pinch down. These equivalence classes may be represented graphically - the location of the earliest chimney being indicated by a node in the corresponding graph. Consider, then, the correlation function $c_{L}(\beta)$ obtained by summing over surfaces $t_{L} \mathrm{CJ}_{L}$ which contain no elementary chimneys other than the first and last:

$$
\begin{gathered}
c_{L}(\beta)=\sum_{S \in t_{L}} e^{-\beta|S|}, \\
t_{L}=\left\{S \in \delta_{L}|| r_{k} \mid>4 \forall k \text { except } k=0, L\right\} .
\end{gathered}
$$

Clearly $c_{L}$ is represented by the graph without nodes. Thus, by analogy to the treatment of the low density Mayer series in [8], we may identify $c_{L}$ as an OrnsteinZernike direct correlation function. According to the ideas of Fisher [9], $c_{L}$ should have a shorter range (i.e., a larger mass) than $q_{\mathrm{L}}$.

Proposition 2.3. For all $\beta$,

$$
\lim _{L \rightarrow \infty} \log c_{L}(\beta) / L \equiv-m_{c}(\beta)
$$

exists. Moreover, for $\beta$ sufficiently large, $m_{c}(\beta)>m(\beta)$.

Proof. That the limit exists follows from another subadditivity estimate of the form

$$
c_{L_{1}+L_{2}}(\beta) \geqq(\text { const }) c_{L_{1}}(\beta) c_{L_{2}}(\beta),
$$

where the constant is independent of the lengths $L_{1}$ and $L_{2}$.

We may bound $m_{c}(\beta)$ below by, say, a Peierls argument which gives

$$
m_{c}(\beta) \geqq 6 \beta \text {-const }
$$

for $\beta$ sufficiently large. Comparing this with the a priori upper bound on $m(\beta)$ given by (2.5), the result is seen to hold for $\beta$ large enough.

Theorem 2.4. Whenever $m_{c}(\beta)>m(\beta)$, there exist constants $\kappa_{1}(\beta), \kappa_{2}(\beta)>0$ such that

$$
\left|q_{L}(\beta) e^{+m(\beta) L}-\kappa_{1}(\beta)\right| \leqq e^{-\kappa_{2}(\beta) L}
$$

uniformly in $L$.

1 The relationship between our bound (2.9) and the upper gap was pointed out to us by J. Fröhlich 
Proof. Partitioning the set $\delta_{L}$ according to the scheme outlined above, it is seen that the contribution from all surfaces which do not pinch down until the $N^{\text {th }}$ step $(2 \leqq N \leqq L-1)$ is given by

$$
\frac{1}{g^{4}} c_{N} q_{L+1-N}, \quad g \equiv e^{-\beta} .
$$

We may therefore write

$$
q_{L}=c_{L}+\frac{1}{g^{4}} \sum_{N=2}^{L-1} c_{N} q_{L+1-N}
$$

or graphically,

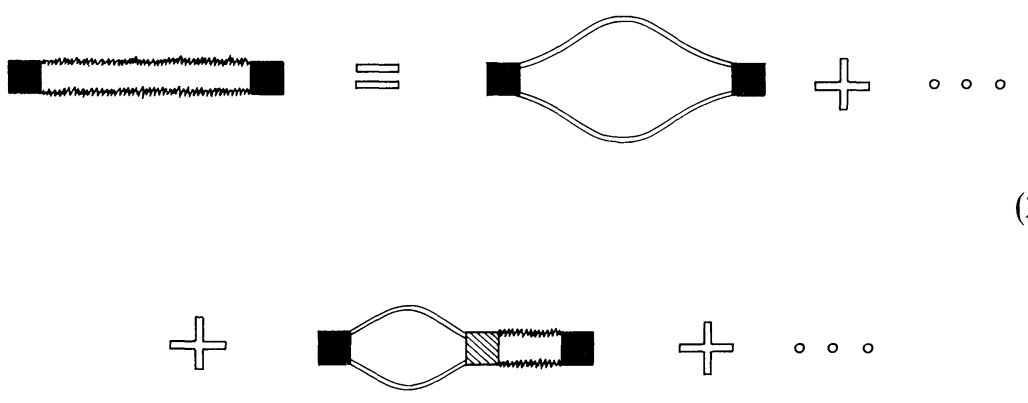

To complete the argument, we will exploit the fact that (2.16) is of form of a convolution. Consider the (discrete) Laplace transform

$$
\hat{q}(z)=\sum_{L} q_{L} z^{L},
$$

with an analogous expression for $\hat{c}(z)$. (Here we define $q_{1}=c_{1}=g^{4}$.) Evidently $\hat{q}(z)$ is analytic in the region $|z|<e^{m}$, while $\hat{c}(z)$ admits the larger region of analyticity $|z|$ $<e^{m_{c}}$. Taking the transform of Eq. (2.16), a little algebra yields

$$
\hat{q}(z)=\frac{z g^{4}}{2-\frac{1}{z g^{4}} \hat{c}(z)}
$$

for $|z|<e^{m}$. Note however that the right-hand side of (2.18) makes sense in the larger region $|z|<e^{m_{c}}$. Indeed, using the bound (2.4), it is easy to show that the function $h(z)=2-\frac{1}{z g^{4}} \hat{c}(z)$ has a simple zero at $z=e^{m}$, and no other zeroes within some larger disk $|z|<e^{m} / \lambda, \lambda<1$. Thus $z g^{4} / h(z)$ defines a meromorphic extension for $\hat{q}(z)$ in the region $|z|<e^{m} / \lambda$ with a simple pole at $z=e^{m}$.

We may therefore write

$$
\hat{q}(z)=F(z) /\left(1-z e^{-m}\right),
$$

with $F(z)$ analytic for $|z|<e^{m} / \lambda$. Noting that $q_{L}$ is simply the coefficient of $z^{L}$ in the expansion of the above expression, we obtain

$$
q_{L} e^{+m L}=\left(F_{0}+F_{1} e^{m}+\ldots+F_{L} e^{m L}\right) .
$$


Finally, recalling the Cauchy bound,

$$
\left|F_{n}\right| \leqq(\text { const }) \lambda^{n} e^{-m n},
$$

the desired result follows easily.

Remark. Equation (2.16) is known as the Ornstein-Zernike equation; it is typically taken as the defining equation for the direct correlation function $c_{L}$. The reader may recognize that the Ornstein-Zernike equation is analogous to the SchwingerDyson equation, which relates the exact propagator to the one-particle irreducible function. This approach has recently been used by Brydges and Spencer [10] in an analysis of the self-avoiding random walk.

ii) Ornstein-Zernike Decay for Unconstrained Tubes. We now treat the case in which the tube is permitted to wander from the $x$-axis. In order to facilitate our analysis, we introduce a generalization of the correlation $Q_{L}(\beta)$, which we denote by $Q_{L,(a, b)}(\beta)$. The latter function is defined by summing over all SOS tubes which begin and end in elementary chimneys, and have as their boundary $p_{0} \cup p_{L,(a, b)}$. Here $p_{L,(a, b)}$ denotes the translate of $p_{L}$ by $a$ units in the $y$ - and $b$ units in the $z$-direction. Thus $Q_{L}=Q_{L,(0,0)}$. (The correlation $Q_{L,(a, b)}$ will be studied in its own right in Sect. 4.) Finally, we also define the "master function," $\mathbb{Q}_{L}$, to be the sum over all SOS tubes which begin at $p_{0}$ and end somewhere in the plane $x=L+1 / 2$, i.e.

$$
\mathbb{Q}_{L}(\beta)=\sum_{a, b} Q_{L,(a, b)}(\beta) .
$$

All of the above have direct correlation counterparts: $C_{L,(a, b)}, C_{L}=C_{L,(0,0)}$ and $\mathbb{C}_{L}$ $=\sum_{a, b} C_{L,(a, b)}$.

As will become apparent, the master functions $\mathbb{Q}_{L}$ and $\mathbb{C}_{L}$ behave similarly to the constrained correlations $q_{L}$ and $c_{L}$ discussed in subsection (i). We shall exploit this analogy to prove the desired scaling for $Q_{L}(\beta)$. First, we note that by the standard subadditivity arguments (cf. Propositions 2.1 and 2.3), the master functions have well-defined masses:

Proposition 2.5. The limits

and

$$
M(\beta) \equiv \lim _{L \rightarrow \infty}\left[-\log \mathbb{Q}_{L}(\beta) / L\right]
$$

exist (in $\mathbb{R}^{*}$ ).

$$
M_{c}(\beta) \equiv \lim _{L \rightarrow \infty}\left[-\log \mathbb{C}_{L}(\beta) / L\right]
$$

Remark. Our use of $M(\beta)$ to denote both the limit in (2.2) and that in (2.23) will be justified by later results (cf. Theorem 2.7).

Next, following the arguments of Eqs. (2.15)-(2.16), we observe that the correlations are related by an Ornstein-Zernike equation:

$$
\begin{gathered}
Q_{L,(a, b)}=C_{L,(a, b)}+\frac{1}{g^{4}} \sum_{N=2}^{L-1} \sum_{a^{\prime}, b^{\prime}} C_{N,(a, b)} Q_{L+1-N,\left(a-a^{\prime}, b-b^{\prime}\right)}, \\
\mathbb{Q}_{L}=\mathbb{C}_{L}+\frac{1}{g^{4}} \sum_{N=2}^{L-1} \mathbb{C}_{N} \mathbb{Q}_{L+1-N} .
\end{gathered}
$$

Equation (2.25B) may be obtained simply by summing (2.25A) over $a$ and $b$. 
The relevant transforms are given by

$$
\begin{gathered}
\hat{Q}_{\beta}\left(z,\left(\omega_{1}, \omega_{2}\right)\right)=\sum_{L, a, b} Q_{L,(a, b)}(\beta) z^{L} e^{i \omega_{1} a} e^{i \omega_{2} b}, \\
\hat{\mathbb{Q}}_{\beta}(z)=\sum_{L} \mathbb{Q}_{L}(\beta) z^{L}=\hat{Q}_{\beta}(z,(0,0)),
\end{gathered}
$$

and similarly for $C$. In the above, $z$ is a complex number (presumably of modulus smaller than $\left.e^{M(\beta)}\right)$, and $-\pi<\omega_{1}, \omega_{2} \leqq \pi$. As before, we use the convolution form of $(2.25 \mathrm{~A})$ to write

$$
\hat{Q}\left(z,\left(\omega_{1}, \omega_{2}\right)\right)=\frac{z g^{4}}{2-\hat{C}\left(z,\left(\omega_{1}, \omega_{2}\right)\right) / z g^{4}} .
$$

The corresponding equation for the master functions is obtained by setting $\omega_{1}=\omega_{2}=0$.

We may regain the quantities $Q_{L}$ by means of the inversion formula

$$
Q_{L}=\frac{1}{2 \pi i} \oint \frac{d z}{z^{L+1}} \int_{-\pi}^{+\pi} d \omega_{1} d \omega_{2} \hat{Q}\left(z,\left(\omega_{1}, \omega_{2}\right)\right)
$$

However, in order to analyze the above integral, we must establish that $\hat{Q}$ and $\hat{C}$ have certain continuity properties. In this context, it is convenient to regard $e^{i \omega_{1}}$ and $e^{i \omega_{2}}$ as complex variables, $\xi_{1}$ and $\xi_{2}$, restricted to the unit circle. Although a weaker result would suffice for the purposes of this section, we show below that for those values of $z$ at which the transformed master functions are regular, the corresponding transformed correlation functions are (separately) regular in $\xi_{1}$ and $\xi_{2}$ in a neighborhood of $\left|\xi_{1}\right|=1$ and $\left|\xi_{2}\right|=1$. We state and prove the lemma for the direct functions; the analogous result also holds for the $Q$ 's.

Lemma 2.6. Take $|z|<e^{M_{c}(\beta)}, \beta>\bar{\beta}$. Then, provided that $\left|\xi_{1}\right|$ and $\left|\xi_{2}\right|$ are sufficiently close to one, the function

$$
\hat{C}_{\beta}\left(z, \xi_{1}, \xi_{2}\right)=\sum_{L, a, b} C_{L,(a, b)}(\beta) z^{L} \xi_{1}^{a} \xi_{2}^{b}
$$

is regular in $\xi_{1}\left(\xi_{2}\right)$ for fixed $\xi_{2}\left(\xi_{1}\right)$.

Proof. If $\left|z_{0}\right|<e^{M_{c}(\beta)}, \beta>\bar{\beta}$, then for $\eta>0$ sufficiently small,

$$
\left|z_{0}\right|<e^{M_{c}(\beta-\eta)} .
$$

(We remind the reader, cf. remark following Proposition 2.2, that $\bar{\beta}$ is the only possible point of discontinuity of $M(\beta)$. Since $M_{c}(\beta) \geqq M(\beta) \forall \beta$, and since $M_{c}(\beta)$ is concave nondecreasing, it is clear that $M_{c}(\beta)$ is continuous whenever $M(\beta)$ is.)

Using the definition of $C_{L,(a, b)}$, we have

$$
\begin{aligned}
C_{L,(a, b)}(\beta-\eta) & =\sum_{S \in \mathscr{T}_{L,(a, b)}} e^{-\beta|S|} e^{+\eta|S|} \\
& \geqq C_{L,(a, b)}(\beta) e^{4 \eta(|a|+|b|)},
\end{aligned}
$$

since each $S \in \mathscr{T}_{L,(a, b)}$ has at least $4(|a|+|b|)$ plaquettes. This implies that $\forall a, b$

$$
C_{L,(a, b)}(\beta-\eta) \geqq C_{L,(a, b)}(\beta)\left|\xi_{1}\right|^{a}\left|\xi_{2}\right|^{b},
$$

provided that $\left|\xi_{1}\right|,\left|\xi_{2}\right|,\left|\xi_{1}\right|^{-1},\left|\xi_{2}\right|^{-1}<e^{4 \eta}$. 
Also note that, for fixed $L$, we have the subadditive bounds

$$
\text { (const) } e^{-M_{c}(\beta-\eta) L} \geqq \mathbb{C}_{L}(\beta-\eta) \equiv \sum_{a, b} C_{L,(a, b)}(\beta-\eta),
$$

where the constant is independent of $L$.

The bounds (2.30), (2.32), and (2.33) imply that the power series (2.29) for $\hat{C}_{\beta}$ is absolutely convergent.

We can now establish the Ornstein-Zernike scaling:

Theorem 2.7. Whenever $M(\beta)<M_{c}(\beta), Q_{L}(\beta)$ has the asymptotic form

$$
Q_{L}(\beta)=\frac{\text { const }}{L} e^{-M(\beta) L}\left[1+O\left(\frac{1}{\sqrt{L}}\right)\right] .
$$

Remark. The standard arguments (cf. Proposition 2.2) show that the hypothesis of the theorem is satisfied for $\beta$ sufficiently large.

Proof. The proof, which reduces to asymptotic analysis of the integral (2.28), is straightforward but tedious. Some of the details have been relegated to Appendix B.

The first step is to demonstrate that the master function $\mathbb{Q}_{L}$ has pure exponential decay in the sense of Eq. (2.9). This is done by following step for step the proof of Theorem 2.4. In particular $\widehat{\mathbb{Q}}(z)$ has a simple pole at $z=e^{M}$ and no other poles in the larger disk $|z|<e^{M} / \lambda$.

Next, a Rouché argument (Lemma B.2) establishes that, when $\omega_{1}$ and $\omega_{2}$ are sufficiently small, the pole structure is similar to that described above. Since $\hat{C}\left(z,\left(\omega_{1}, \omega_{2}\right)\right)$ is smooth (indeed analytic) in $\left(\omega_{1}, \omega_{2}\right)$, the implicit function theorem may be invoked to show that there is a function $e^{M\left(\omega_{1}, \omega_{2}\right)}$ which describes the motion of the simple pole for small $\left(\omega_{1}, \omega_{2}\right)$. This pole is, of course, the principal contribution to the integral (2.28) for $\left(\omega_{1}, \omega_{2}\right)$ sufficiently small. Finally, a detailed argument (Lemma B.1) shows that the relative contribution to the integral from those $\left(\omega_{1}, \omega_{2}\right)$ outside any neighborhood of the origin is exponentially small. We therefore have

$$
Q_{L}(\beta)=\int_{\left|\omega_{1}\right|,\left|\omega_{2}\right|<\delta} d \omega_{1} d \omega_{2} e^{-L M\left(\omega_{1}, \omega_{2}\right)} F\left(\omega_{1}, \omega_{2}\right)\left[1+O\left(e^{-\varepsilon L}\right)\right] .
$$

In the above, $\delta$ is some suitably chosen constant which ensures that both the Rouche argument in Lemma B.2 and the use of the implicit function theorem are legitimate. The function $F\left(\omega_{1}, \omega_{2}\right)$ is continuous in $\left(\omega_{1}, \omega_{2}\right)$ and independent of $L$. Because all the coefficients $C_{L,(a, b)}$ are nonnegative and symmetric in $a \leftrightarrow-a$, $b \leftrightarrow-b$, it follows that $e^{M\left(\omega_{1}, \omega_{2}\right)}$ has a quadratic minimum, i.e.

$$
e^{M\left(\omega_{1}, \omega_{2}\right)}=e^{M+\gamma\left(\omega_{1}^{2}+\omega_{2}^{2}\right)+\ldots} .
$$

(That the coefficients of $\omega_{1}^{2}$ and $\omega_{2}^{2}$ are identical follows from the $a \leftrightarrow b$ symmetry.) The standard asymptotic analysis (e.g., Laplace's method) may now be applied to the $\omega$ integrations, from which the desired result follows easily.

Remark. The analysis above is easily extended to SOS tubes in higher dimensions. In general, the class $\mathscr{S}_{L}^{\prime}$ of tubes is characterized by the constraint that in each 
subspace $x=n+1 / 2$, there is a single (self-avoiding) ring of plaquettes. The plaquette rings in successive subspaces must be spanned by a (self-avoiding, orientable) surface in the intervening hyperspace. Results similar to those derived in this section, as well as the generalization of Theorem 4.1, can then be obtained for $d \geqq 4$. It is easy to see that each extra dimension provides an additional transverse degree of freedom, and thus another factor of $1 / \sqrt{L}$ in the generalization of Theorem 2.7. One must of course modify the constants in the bounds which guarantee a region where $M(\beta)<M_{c}(\beta)$.

(iii) Monotone Approximants to the Mass. Here we outline a simple procedure for obtaining upper bounds on the mass of the pair correlation function. The procedure involves little more than an observation on the structure of the transformed Ornstein-Zernike equation; nevertheless, it seems to be quite useful computationally. We note that the Ornstein-Zernike equation (2.27) is valid whenever $|z|<e^{M(\beta)}$, regardless of whether $M_{c}(\beta)>M(\beta)$. [Although we use the master function notation of subsection (ii), the results apply equally well to constrained tubes.]

Let $\mathbb{\mathbb { D }}(z)$ denote a power series, $\hat{\mathbb{D}}(z)=\sum_{L} \mathbb{D}_{L} z^{L}$, with coefficients satisfying $\mathbb{C}_{L}$ $\geqq \mathbb{D}_{L} \geqq 0$. In particular, $\hat{\mathbb{D}}(z)$ may be obtained by summing over a subclass of the tubes contributing to $\widehat{\mathbb{C}}(z)$. Comparing the expression

$$
\widehat{\mathbb{G}}(z)=\frac{z g^{4}}{2-\frac{1}{z g^{4}} \hat{\mathbb{D}}(z)} \equiv \sum_{L} \mathbb{G}_{L} z^{L}
$$

to the transformed Ornstein-Zernike equation (2.27), it is easy to see that $\mathbb{G}_{L}$ $\leqq \mathbb{Q}_{L} \forall L$. Thus, if the chosen $\hat{\mathbb{D}}(z)$ is such that the first (real, positive) zero of $2-\frac{1}{z g^{4}} \hat{\mathbb{D}}(z)$ is simple, then this zero provides an upper bound on $e^{M(\beta)}$. It is easily seen that the bound improves as one enlarges the subclass of tubes defining $\hat{\mathbb{D}}(z)$.

\section{SOS Pair Correlations for Percolation}

In this section, we generalize some of the results of the preceding section to an ensemble of Bernoulli plaquettes in $d=3$. The relevant plaquette events will be the formation of open (i.e., unobstructed) SOS tubes which span a preprescribed pair of loops. The study of such events is motivated by the percolation analogue of the glue-ball propagator:

$$
\left\langle W_{\gamma_{1} \gamma_{2}}\right\rangle_{p}-\left\langle W_{\gamma_{1}} \cap W_{\gamma_{2}}\right\rangle_{p}
$$

In the above expression, $\gamma_{1}$ and $\gamma_{2}$ are planar loops composed of lattice edges, $W_{\gamma_{1} \ldots \gamma_{k}}$ is the event that there is a set of occupied plaquettes with boundary $\gamma_{1} \cup \ldots \cup \gamma_{k}$, and $\langle-\rangle_{p}$ denotes the probability with respect to Bernoulli measure at plaquette density $p$. Expressing the above quantity as the expectation of the difference of characteristic functions, it is not difficult to see that the only configurations which contribute are those in which the pair of loops, $\gamma_{1}$ and $\gamma_{2}$, form the boundary of an unobstructed tube. This is most easily visualized in $d=3$ 


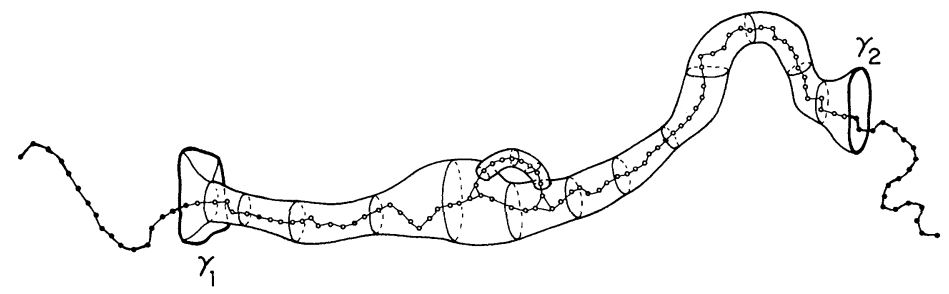

Fig. 2. A configuration in $W_{\gamma_{1} \gamma_{2}} \backslash W_{\gamma_{1}} \cap W_{\gamma_{2}}$

where the event that a given plaquette is unoccupied is equivalent to the occurrence of an occupied (dual) bond. The expression (3.1) is then the probability of the event depicted in Fig. 2.

Here we study what we believe to be a representative subset of the above event, namely when (at least one of) the spanning tubes is of the SOS type.

It should be noted that the study of these objects is not only of interest in the context of percolation. As a model of a correlation function, quantities of this form do not suffer from the (inevitable) catastrophic transitions which plague models of the type described in Sect. 2. This is due to the fact that we are dealing with probabilities, and thus all "masses" will be bounded below by zero. In this sense, correlation functions of the type defined here provide a better forum for the study of phase transitions in realistic systems.

The remainder of this section is devoted to defining the relevant events and showing that an Ornstein-Zernike equation is satisfied by the corresponding probabilities. The scaling (1.2) then follows from an analysis similar to that of the previous section.

For clarity of exposition, we begin, as in Sect. 2, with the case of constrained SOS tubes. Let $\Omega_{L}$ denote the $\sigma$-algebra of plaquette events in the strip $0<x$ $<L+1$. As before, we consider plaquettes on the dual of the cubic lattice $\mathbb{Z}^{3}$. Thus to each unoccupied plaquette, there corresponds an occupied bond of $\mathbb{Z}^{3}$. We define $\phi_{L}^{\prime} \subset \Omega_{L}$ to be the event that:

(i) $p_{0} \cup p_{L}$ is the boundary of a constrained SOS tube composed of occupied plaquettes; and

(ii) the origin is connected to the point $(L+1,0,0)$ by a path of occupied bonds.

Remark. Since $\phi_{L}^{\prime} \subset \Omega_{L}$, both the plaquettes and the bonds referred to above must lie entirely within the strip $0<x<L+1$. In particular, this means that the path of occupied bonds cannot include any bonds from the planes $x=0$ and $x=L+1$. Thus the existence of the tube forces the path of occupied bonds to lie inside of it.

The above definition may be written more formally as

$$
q_{L}^{\prime}=\left\{\omega \in \Omega_{L} \mid \exists S \in \jmath_{L}^{\prime}, S \text { occupied; }(0,0,0) \in \text { C.C. }((L+1,0,0))\right\},
$$

where $\sigma_{L}^{\prime}$ is the class of surfaces defined in Sect.2, and C.C. $(r)$ denotes the connected bond cluster of the point $r \in \mathbb{Z}^{3}$. We remind the reader that the prime on $\sigma_{L}^{\prime}$ denotes the fact that the surfaces need not begin and end with elementary chimneys.

Since events in non-intersecting strips are disjoint, we obtain the subadditive estimate:

$$
\left\langle q_{L_{1}+L_{2}+1}^{\prime}\right\rangle_{p} \geqq p^{4}\left\langle q_{L_{1}}^{\prime}\right\rangle_{p}\left\langle\phi_{L_{2}}^{\prime}\right\rangle_{p}
$$


The inequality follows from the fact that we have only constructed a subset of $q_{L_{1}+L_{2}+1}^{\prime}$ by joining the two tubes in the simplest fashion. Note that the above relation cannot be obtained by invoking the FKG inequality [11, 12], since the events $\phi_{L}^{\prime}$ are neither positive nor negative.

Proposition 3.1. For all $p \in[0,1]$, there exists

$$
m(p) \equiv-\lim _{L \rightarrow \infty} \log \left\langle q_{L}^{\prime}\right\rangle_{p} / L \geqq 0 .
$$

Proof. Existence is a consequence of (3.3). The lower bound follows from the fact that $\left\langle q_{L}^{\prime}\right\rangle_{p}$ is a probability.

For a given $\omega \in \phi_{L}^{\prime}$ there are, in general, many surfaces $S \in \delta_{L}^{\prime}$ which are occupied. The following proposition shows that it is possible to uniquely select one of these tubes.

Proposition 3.2. There exists a function $S: q_{L}^{\prime} \rightarrow J_{L}^{\prime}$.

Proof. Given two (contrained) SOS tubes $S_{1}, S_{2} \in \sigma_{L}^{\prime}$, it is possible to construct a third tube out of some of their plaquettes. This is done by first regarding $S_{1}$ and $S_{2}$ as the boundaries of geometric solids $S_{1}^{(i)}$ and $S_{2}^{(i)}$, and then taking the boundary of the solid which is the result of their intersection: $S=\partial\left(S_{1}^{(i)} \cap S_{2}^{(i)}\right)$. Since both $S_{1}$ and $S_{2}$ surround the $x$-axis, it is clear that $S \in \sigma_{L}^{\prime}$, and that all the plaquettes in $S$ are in either $S_{1}$ or $S_{2}$. Then

Let $\omega \in q_{L}^{\prime}$, and denote by $S_{1}, \ldots, S_{k}, \ldots$ all tubes in $\delta_{L}^{\prime}$ which are occupied in $\omega$.

$$
S(\omega) \equiv \partial\left(S_{1}^{(i)} \cap \ldots \cap S_{k}^{(i)} \cap \ldots\right) \in \delta_{L}^{\prime}
$$

is also occupied.

Remarks. (1) Note that $S(\omega)$ is the minimal SOS tube in the configuration $\omega \in q_{L}^{\prime}$.

(2) For unconstrained tubes (which need not have an axis in common) the above procedure would in general fail to produce any tube at all. However, we are concerned with tubes which enclose a path of occupied bonds. Indeed, since all occupied tubes in a given configuration must contain the connected bond cluster of the origin, their intersection will produce a (unique) SOS tube.

The function defined in Proposition 3.2 allows us to associate disjoint events with distinct surfaces $S \in \delta_{L}^{\prime}$. We shall use the rather abusive, but suggestive notation $S \subset \Omega_{L}$ to denote the event

$$
S=\left\{\omega \in q_{L}^{\prime} \mid S(\omega)=S\right\} .
$$

This allows us to partition the event $\phi_{L}^{\prime}$. In particular, it suggests the following definition of the direct correlation event:

$$
c_{L}^{\prime}=\left\{\omega \in q_{L}^{\prime} \mid S(\omega) \in t_{L}^{\prime}\right\}
$$

(Here $t_{L}^{\prime}$ is the set of all constrained SOS tubes which have no elementary chimneys.)

As in the previous section, we find it convenient to introduce analogues of $q_{L}^{\prime}$ and $c_{L}^{\prime}$ for which the SOS surface must begin and end in an elementary chimney. To this end, let $\operatorname{ch}(\cdot)$ denote the event that the four plaquettes which comprise the 
elementary chimney surrounding the point $(\cdot, 0,0)$ are occupied. The "unprimed" events are defined by

$$
\begin{aligned}
q_{L+2} & =\operatorname{ch}(0) \cup q_{L}^{\prime} \cup \operatorname{ch}(L+1), \\
c_{L+2} & =\operatorname{ch}(0) \cup c_{L}^{\prime} \cup \operatorname{ch}(L+1) .
\end{aligned}
$$

Note that the above quantities are elements of the $\sigma$-algebra of plaquette events in the strip $-1 / 2<x<L+3 / 2$, since $\operatorname{ch}(\xi)$ consists of plaquette events in $\xi-1 / 2<x$ $<\xi+1 / 2$. Since the three events in each of the definitions (3.8) occur in disjoint regions, we have

$$
\begin{aligned}
\left\langle q_{L+2}\right\rangle_{p} & =p^{8}\left\langle q_{L}^{\prime}\right\rangle_{p}, \\
\left\langle c_{L+2}\right\rangle_{p} & =p^{8}\left\langle c_{L}^{\prime}\right\rangle_{p} .
\end{aligned}
$$

Let $T^{N}$ denote the operator for translation by $N$ units in the $x$-direction. It is not difficult to see that $q_{L+2}$ may be expressed as the disjoint union

$$
q_{L+2}=c_{L+2} \cup\left[\bigcup_{N=0}^{L-3} c_{N+2} \cup T^{N+1}\left(q_{L-(N+1)}^{\prime}\right) \cup \operatorname{ch}(L+1)\right],
$$

so that, by (3.9a) and a shift of indices,

$$
\left\langle q_{L}\right\rangle_{p}=\left\langle c_{L}\right\rangle_{p}+\frac{1}{p^{4}} \sum_{N=2}^{L-1}\left\langle c_{N}\right\rangle_{p}\left\langle q_{L-N+1}\right\rangle_{p} .
$$

To proceed with the analysis, we must establish the analogue of Proposition 2.3, namely:

Proposition 3.3. For all $p \in[0,1]$, there exists

$$
m_{c}(p) \equiv-\lim _{L \rightarrow \infty} \log \left\langle c_{L}\right\rangle_{p} / L \geqq 0 .
$$

Furthermore, for $p$ sufficiently small, $m_{c}(p)>m(p)$.

Proof. Existence of the limit follows from a subadditivity estimate of the form

$$
\left\langle c_{L_{1}+L_{2}+1}\right\rangle_{p} \geqq f(p)\left\langle c_{L_{1}}\right\rangle_{p}\left\langle c_{L_{2}}\right\rangle_{p},
$$

which (as before) requires a construction to guarantee that the tubes join in a nodeless fashion. However, this estimate requires somewhat more caution than that of Eq. (2.13). Here, we begin with a tube in $c_{L_{1}}^{\prime}$ and another in $T^{L_{1}+1}\left(c_{L_{2}}^{\prime}\right)$, which are related to the unprimed events by Eq. (3.9b). The crucial point for the derivation of (3.13) is that the nature of plaquette events in the initial and final planes of a given tube is, to some extent, determined. In particular, since the minimal SOS tube in some partition of $c_{L}^{\prime}$ can have no elementary chimneys, at least one of the plaquettes adjacent to each of the boundary plaquettes, $p_{0}$ and $p_{L}$, must be occupied. Since these plaquettes are guaranteed to be present, we can remove any of them at the expense of a factor of $p$ (i.e., there is no inequality incurred by conditioning problems). If this is done in the final plane of the first tube and the initial plane of the second tube, then it is possible to patch the two tubes together in some (standard) fashion, in such a way that the resulting minimal tube is nodeless. With such a prescription, the subadditivity (3.13) is straightforward. 
In order to demonstrate the existence of a region of $p$ for which the masses are separated, we first note the analogue of the corollary to Proposition 2.1:

$$
e^{-m(p)} \geqq(1-p) p^{4} \quad \forall p \in[0,1] .
$$

(The factor of $(1-p)$ arises from the internal bonds.) To bound $m_{c}(p)$ from below, we use the notation (3.6) to write $\left\langle c_{L}\right\rangle_{p}$ in the form

$$
\left\langle c_{L}\right\rangle_{p}=\sum_{S \in t_{L}}\langle S\rangle_{p} .
$$

However $\langle S\rangle_{p}$, which denotes the probability that $S$ is the minimal occupied tube and that $S$ contains a path of bonds, is clearly bounded above by $p^{|S|}$, the probability that $S$ is occupied. Thus, for $p=e^{-\beta}$,

$$
e^{-m_{c}(p)} \leqq e^{-m_{c}(\beta)},
$$

where $m_{c}$ is the direct correlation mass introduced in (2.12). For $p$ sufficiently small, the lower bound (3.16) on $m_{c}(p)$ exceeds the upper bound (3.14) on $m(p)$.

The proof of scaling for constrained SOS correlations in percolation is identical to that of Theorem 2.4, with the result:

Theorem 3.4. Whenever $m_{c}(p)>m(p)$, there exist constants $k_{1}(p), k_{2}(p)>0$ such that

$$
\left|\left\langle q_{L}\right\rangle_{p} e^{+m(p) L}-k_{1}(p)\right| \leqq e^{-k_{2}(p)}
$$

uniformly in $L$.

We now turn to an investigation of unconstrained tube correlations for the Bernoulli ensemble. Our analysis will parallel that of Sect. 2(ii). First, we define the unconstrained pair correlation event $\mathscr{Q}_{L,(a, b)}$ by analogy to $q_{L}$, replacing the set of surfaces $\jmath_{L}$ by the unconstrained tubes $\mathscr{\mathscr { S }}_{L,(a, b)}$. The relevant transforms are

$$
\begin{gathered}
\hat{Q}_{p}\left(z,\left(\omega_{1}, \omega_{2}\right)\right)=\sum_{L, a, b}\left\langle\mathscr{Q}_{L,(a, b)}\right\rangle_{p} z^{L} e^{i \omega_{1} a} e^{i \omega_{2} b}, \\
\hat{\mathbb{Q}}_{p}(z) \equiv \hat{Q}_{p}(z,(0,0)),
\end{gathered}
$$

which, of course, have direct correlation function analogues. [We have taken the liberty of using notation identical to that of Sect. 2(ii), so that the results of Appendix B may be easily applied to either system.] As before (cf. Proposition 2.5), it can be shown that the master functions $\widehat{\mathbb{Q}}$ and $\widehat{\mathbb{C}}$ have well-defined masses which we denote by $M(p)$ and $M_{c}(p)$, respectively. Furthermore, the reasoning used in previous derivations shows that $\hat{Q}$ and $\hat{C}$ are related by the Ornstein-Zernike equation (2.27), with $g$ replaced by $p$.

A key ingredient in the proof of Ornstein-Zernike scaling of $Q_{L}(\beta)$ was Lemma 2.6 which established smoothness of the transforms in the variables $\omega_{1}$ and $\omega_{2}$. Since no obvious analogue of the technique used to prove Lemma 2.6 applies to the Bernoulli system, we follow an alternative approach which exploits decay of percolation connectivity functions. We begin by defining a critical point which plays a central role in our analysis. 
Definition. Let $E$ be an edge of the (dual) lattice $\mathbb{Z}^{3 *}$. Denote by C.P.C.(E) the connected plaquette cluster ${ }^{2}$ of the edge $E$ and let $\mid$ C.P.C. $(E) \mid$ denote its size. The critical clustering point, $\sigma_{c}$, is given by

$$
\sigma_{c}=\sup \left\{p \mid\langle| \text { C.P.C. }(E)|\rangle_{p}<\infty\right\} \text {. }
$$

Remark. The point $\sigma_{c}$ should be distinguished from other critical points of the Bernoulli system. Although it is probable that $\sigma_{c}$ coincides with the percolation threshold for plaquettes (that is, the critical point for the formation of infinite clusters of plaquettes), $\sigma_{c}$ is unrelated to the critical point for the formation of infinite sheets which was analyzed in [4]. Indeed, the latter critical point occurs (in $d=3$ ) at a plaquette density strictly larger than $1 / 2$, while an easy application of the Kesten method [13] demonstrates that $\sigma_{c} \leqq 1 / 2$ for all $d \geqq 3$. The relationship between $\sigma_{c}$ and the Curie point in a certain type of spin system is discussed in [14].

Definition. Let $E_{1}$ and $E_{2}$ be edges of the (dual) lattice $\mathbb{Z}^{3^{*}}$ with coordinates $\left(x_{1}, y_{1}, z_{1}\right)$ and $\left(x_{2}, y_{2}, z_{2}\right)$, respectively. Denote by $\left|E_{1}-E_{2}\right|=\left|x_{1}-x_{2}\right|+\left|y_{1}-y_{2}\right|$ $+\left|z_{1}-z_{2}\right|$ the $\ell^{\infty}$ distance between $E_{1}$ and $E_{2}$. The connectivity function $\Lambda_{E_{1}, E_{2}}$ is the probability that $E_{1}$ and $E_{2}$ are connected by occupied plaquettes, i.e. the probability of the event $\left\{E_{1} \in\right.$ C.P.C. $\left.\left(E_{2}\right)\right\}$.

In order to prove the analogue of Lemma 2.6, we shall need the following proposition, which describes the decay of the connectivity function.

Proposition 3.5. Whenever $p<\sigma_{c}$, there exists a positive constant $\lambda$ such that

$$
\Lambda_{E_{1}, E_{2}} \leqq e^{-\lambda\left|E_{1}-E_{2}\right|}
$$

for $\left|E_{1}-E_{2}\right|$ sufficiently large.

Proposition 3.5 is by now a standard result in percolation theory. It was first derived in a general context by Kesten [15]. Alternative derivations using rescaling methods and/or inequalities of the Lieb-Simon type [16, 17] may be found in $[4,18,19]$.

Lemma 3.6. Let $p<\sigma_{c}$ and take $|z|<e^{M(p)}\left[|z|<e^{M_{c}(p)}\right]$. Then in a neighborhood of $\left|\xi_{1}\right|=1$ and $\left|\xi_{2}\right|=1$, the transformed correlation function $\hat{Q}_{p}\left(z, \xi_{1}, \xi_{2}\right)\left[\hat{C}_{p}\left(z, \xi_{1}, \xi_{2}\right)\right]$ is (separately) regular in $\xi_{1}$ and $\xi_{2}$.

The proof of Lemma 3.6 is rather involved and has been relegated to Appendix A. Although the cornerstone is clearly Proposition 3.5, we also use certain properties of decay constants which are developed along the lines of the analysis in Sect. 5.

We may now follow the reasoning of Theorem 2.7 (and Appendix B) mutatis mutandis, with the result:

Theorem 3.7. If $p<\sigma_{c}$, and in addition $M(p)<M_{c}(p)$, then

$$
\left\langle\mathscr{Q}_{L}\right\rangle_{p}=\frac{\text { const }}{L} e^{-M(p)}\left[1+O\left(\frac{1}{\sqrt{L}}\right)\right] \text {. }
$$

2 We remind the reader that two plaquettes are said to be connected if they have an edge in common. The connected plaquette cluster C.P.C. $(E)$ is composed of occupied plaquettes connected to each other, at least one of which has $E$ in its boundary 


\section{Properties of Typical Tubes}

(i) Wandering of Tubes. In this section, we investigate some structural properties of typical SOS tubes. We first determine the distribution of endpoints in the plane $x=L$ (i.e., the ratio $\left.Q_{L,(a, b)} / \mathbb{Q}_{L}\right)$, which indicates how far the typical tube wanders.

The results of Theorems 2.7 and 3.7, which show that whenever $M(\beta)<M_{c}(\beta)$ (or $M(p)<M_{c}(p)$ with $p<\sigma_{c}$ )

$$
Q_{L} \sim A \frac{1}{\alpha \pi L} e^{-M L} \quad \text { and } \quad \mathbb{Q}_{L} \sim A e^{-M L},
$$

strongly suggest that the motion of the tubes is normally distributed. That this is the case is established in the next theorem.

Theorem 4.1. Whenever $M(\beta)<M_{c}(\beta)\left(\right.$ or $M(p)<M_{c}(p)$ with $\left.p<\sigma_{c}\right)$, the asymptotic behavior of $Q_{L,(a, b)}$ is given by

$$
Q_{L,(a, b)}=\frac{1}{\pi \alpha L} e^{-M L} e^{-\left[\left(a^{2}+b^{2}\right) / \alpha L\right]}\left[1+O\left(\frac{1}{\sqrt{L}}\right)\right] .
$$

Proof. By the analsis in Appendix B, we have the following generalization of Eq. (2.35):

$$
Q_{L,(a, b)}=\int_{\left|\omega_{1}\right|,\left|\omega_{2}\right|<\delta} e^{-M\left(\omega_{1}, \omega_{2}\right) L} e^{-i \omega_{1} a} e^{-i \omega_{2} b} F\left(\omega_{1}, \omega_{2}\right)\left[1+O\left(e^{-\varepsilon L}\right)\right] .
$$

Now we simply expand $M\left(\omega_{1}, \omega_{2}\right)$ about its quadratic minimum and complete the square in the exponent. (Note that this is equivalent to a distortion of the contour in the $\omega_{1}, \omega_{2}$ planes, which requires the regularity established in Lemma 2.6 and Appendix A.) The result now follows from standard asymptotic analysis (e.g., the method of steepest descents).

Comparing the above result with the asymptotic behavior of $\mathbb{Q}_{L}$, and using the usual scaling $a=a / \sqrt{L}, b=b / \sqrt{L}$, we obtain a normal distribution

$$
\varrho(a, b)=\frac{1}{\pi \alpha} e^{-\left(a^{2}+b^{2}\right) / \alpha},
$$

indicative of uncoupled free particle motion in the perpendicular planes. Evidently, a typical tube in $\mathscr{S}_{L}$ wanders a distance $\sim \sqrt{L}$.

(ii) Stability of Constrained Tubes. A more subtle question than that of wandering is whether the typical tube remains of finite width as $L \rightarrow \infty$. In this subsection, we investigate the width of typical tubes for free random surface models of the form (1.1). [See Subsect. (iii) for analogous results in percolation.] In order to uncouple this question from that of wandering, we restrict attention to constrained SOS tubes.

Let $\mathbf{r}_{h}$ denote a point in the plane $x=L+1 / 2$, a distance $h$ from the $x$-axis. We consider the probability, in the ensemble of constrained tubes, that the point $\mathbf{r}_{h}$ is enclosed by a surface $S \in \Im_{2 L+1}$ :

$$
\mathscr{P}_{L}(h ; \beta)=\sum_{S \in \Delta_{2 L+1}} e^{-\beta|S|} \chi\left(\mathbf{r}_{h} \in \operatorname{Int}(S)\right) / q_{2 L+1}(\beta) .
$$




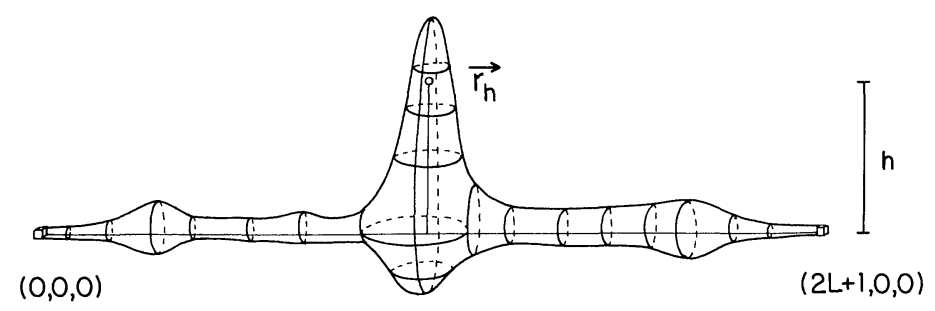

Fig. 3. A surface $S$ which encloses $\mathbf{r}_{h}$

In the above expression, $\chi\left(\mathbf{r}_{h} \in \operatorname{Int}(S)\right)$ is the characteristic function of the event, depicted in Fig. 3, that $\mathbf{r}_{h}$ is enclosed by the surface $S$.

Our criterion for the width of typical tubes is as follows: If

$$
\limsup _{h \rightarrow \infty} \limsup _{L \rightarrow \infty} \mathscr{P}_{L}(h ; \beta)=0,
$$

the tubes are said to be stable; while if

$$
\liminf _{L \rightarrow \infty} \mathscr{P}_{L}(h ; \beta)>0 \text { uniformly in } h,
$$

the tubes are said to breathe. A transition from the former behavior to the latter at some finite $\beta_{b}>\beta_{c}$ will be called a breathing transition.

This transition is, in some sense, the analogue of the roughening transition for the Wilson loop variable. In both cases, the transitions indicate a qualitative change in the structure of contributing surfaces. There is some evidence [20] that the corrections to exponential decay of the Wilson loop variable are altered when a system undergoes a roughening transition. Similarly, one might expect a change in the corrections to exponential decay of the pair correlation for a system which undergoes a breathing transition. Indeed, our proof of Ornstein-Zernike scaling lends some credence to this expectation. If a breathing transition were to occur, the surfaces contributing to $q_{L}$ would typically be quite wide, and thus would resemble those contributing to $c_{L}$. We would therefore expect the masses $m(\beta)$ and $m_{c}(\beta)$ to coincide beyond a breathing transition. Recall that our proof of OrnsteinZernike scaling depended on separation of the masses. While it is possible that such a condition is not necessary, a comparison with exact solutions in two dimensions indicates that separation of the masses is not merely an artifact of our proof. If one examines the exact solution for the two-dimensional Ising pair correlation [21], it is seen that (1) $m(\beta)=m_{c}(\beta) \forall \beta<\infty$, (2) the system satisfies our breathing criterion (4.7) for all nonzero temperatures, and (3) the power law correction to exponential decay of the pair correlation is dramatically different than that predicted by Ornstein-Zernike theory. Our mass separation criterion is also analogous to that for the transition from partial to complete wetting in an exactly solvable planar Ising model [22].

In the remainder of this subsection, we shall show that constrained SOS tubes are stable (i.e., of finite expected width) whenever $\beta$ is larger than the melting point of a related two-dimensional model. This result is analogous to that of van Beijeren [23] on the stability of interfaces in the three-dimensional Ising magnet. 
We begin by defining a related model for the behavior of a rings in a plane. Let $\mathscr{R}_{0}$ be the set of all self-avoiding rings surrounding the origin on the lattice $\mathbb{Z}^{2}$. The partition function of this system is given by

$$
z_{0}(\beta)=\sum_{r \in \mathscr{R}_{0}} e^{-\beta|r|},
$$

where $|r|$ denotes the perimeter of the ring $r \in \mathscr{R}_{0}$. Alternatively, $z_{0}(\beta)$ may be written in the form

$$
z_{0}(\beta)=\sum_{\ell \geqq 4} \varrho(\ell) e^{-\beta \ell},
$$

where $\varrho(\ell)$ is the number of rings $r \in \mathscr{R}_{0}$ of perimeter $\ell$.

Proposition 4.2. The limit $\lim _{L \rightarrow \infty} \log \varrho(\ell) / \ell$ exists.

Proof. This follows from subadditivity. First, let $\varrho(\ell)$ denote the number of distinct rings $r \in \mathscr{R}_{0}$ (i.e., the number of rings in $\mathscr{R}_{0}$ which cannot be obtained from one another by translation). Clearly

$$
\text { (const) } \ell \varrho(\ell) \leqq \varrho(\ell) \leqq(\text { const }) \ell^{2} \varrho(\ell) \text {. }
$$

Now a simple construction shows that

$$
\varrho\left(\ell_{1}+\ell_{2}\right) \geqq \varrho\left(\ell_{1}\right) \varrho\left(\ell_{2}\right),
$$

from which the desired result follows easily.

Remark. The above proposition establishes a critical point for the melting of rings in the plane:

$$
\bar{\beta}^{(2)} \equiv \lim _{L \rightarrow \infty} \log \varrho(\ell) / \ell .
$$

Standard arguments show that $0<\bar{\beta}^{(2)}<\infty$. For future reference, we note that whenever $\beta>\bar{\beta}^{(2)}$ : (1) all the moments of (4.9) are finite, i.e.,

$$
\sum_{\ell} \ell^{k} \varrho(\ell) e^{-\beta \ell}<\infty
$$

and (2) the tail of the sum (4.9) decays exponentially, i.e.

$$
\sum_{\ell>T} \varrho(\ell) e^{-\beta \ell} \leqq(\text { const }) e^{-\left(\beta-\bar{\beta}^{(2)}\right) T} .
$$

Our principal result is the following:

Theorem 4.3. Whenever $\beta>\bar{\beta}^{(2)}$, there exists a $\lambda(\beta)>0$ such that

$$
\limsup _{L \rightarrow \infty} \mathscr{P}_{L}(h ; \beta) \leqq(\text { const }) e^{-\lambda(\beta) h} .
$$

In order to prove the above theorem, we introduce yet another random surface pair correlation. Let $u_{L}$ denote the set of all constrained SOS tubes which begin with an elementary chimney on $p_{0}$, but end in an arbitrary ring in the plane $x=L+1 / 2$. In other words, we have taken the set $\delta_{L}$ and relaxed the constraint that the tubes close at one of the ends. Defining the open pair correlation

$$
o_{L}=\sum_{S \in u_{L}} e^{-\beta|S|}
$$


we have the obvious bound

$$
o_{L} \geqq q_{L} .
$$

Heuristically, one expects that if the tubes do not breathe, then the typical tube contributing to $o_{L}$ should end in a ring of finite width. If this is the case, then $o_{L}$ and $q_{L}$ should not differ significantly, so that (4.17) should be supplemented with a bound of the opposite type. That this occurs for $\beta>\bar{\beta}^{(2)}$ is the content of the following lemma.

Lemma 4.4. Whenever $\beta>\bar{\beta}^{(2)}$, there exists a constant $\delta(\beta)>0$ such that

$$
q_{L} \geqq \delta(\beta) o_{L-1}
$$

uniformly in $L$.

Proof. Observe that we may obtain any surface in $\jmath_{L}$ by taking a surface in $\varkappa_{L-1}$, plating the final ring with vertical plaquettes and adding a chimney. The strategy is to show that the final plating is (almost surely) not too costly.

To this end, let $P(S)$ denote the number of plaquettes required to plate the final ring of $S \in \varkappa_{L-1}$. We have

$$
q_{L}=\sum_{S \in u_{L-1}} e^{-\beta|S|} e^{-\beta P(S)} e^{-4 \beta} .
$$

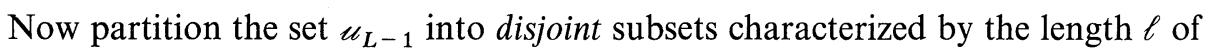
the final ring:

Then

$$
u_{L-1}=\bigcup_{\ell} u_{L-1}(\ell) \text {. }
$$

$$
\sum_{S \in u_{L}-1} e^{-\beta|S|} e^{-\beta P(S)} \geqq \sum_{\ell} e^{-\beta a \ell^{2}} \sum_{S \in u_{L}-1(\ell)} e^{-\beta|S|},
$$

where $a=$ const. Defining the (normalized) weights

$$
w_{\ell}=\sum_{S \in u_{L-1}(\ell)} e^{-\beta|S|} / o_{L-1}
$$

we have, by Eqs. (4.19) and (4.21),

$$
q_{L} \geqq e^{-4 \beta} o_{L-1} \sum_{\ell} w_{\ell} e^{-\beta a \ell^{2}} .
$$

Next, we obtain an upper bound on the $w_{\ell}$. To do this, note that any surface in $u_{L-1}(\ell)$ may be constructed by choosing an appropriate surface $u_{L-2}$, adding a ring of length $\ell$, and plating the (symmetric) difference. Thus

$$
\begin{aligned}
o_{L-1} w_{\ell} & =\sum_{S \in u_{L-1}} e^{-\beta|S|} \sum_{r \in \mathscr{R}_{0}(\ell)} e^{-\beta|r|} e^{-\beta P(S, r)} \\
& \leqq \sum_{S \in u_{L-2}} e^{-\beta|S|} e^{-\beta \ell} \sum_{r \in \mathscr{R}_{0}(\ell)}=o_{L-2} e^{-\beta \ell} \varrho(\ell),
\end{aligned}
$$

where the inequality has been obtained by neglecting the "plating factor" $P(S, r)$. We also observe that adding a chimney to the left end of a tube in $u_{L-2}$ certainly gives us a (translate of a) tube in $u_{L-1}$, so that

$$
o_{L-1} \geqq e^{-4 \beta} o_{L-2} .
$$


Combining this with Eq. (4.24), we have

$$
w_{\ell} \leqq e^{4 \beta} e^{-\beta \ell} \varrho(\ell) \text {. }
$$

Finally, we apply Jensen's inequality to the normalized sum in (4.23), and use the bound (4.26) to obtain

$$
\begin{aligned}
q_{L} & \geqq e^{-4 \beta} o_{L-1} \exp \left(-a \beta \sum_{\ell} \ell^{2} w_{\ell}\right) \\
& \geqq e^{-4 \beta} \exp \left(-a \beta e^{4 \beta} \sum_{\ell} \ell^{2} e^{-\beta \ell} \varrho(\ell)\right) o_{L-1} \equiv \delta(\beta) o_{L-1} .
\end{aligned}
$$

Since $\beta>\bar{\beta}^{(2)}$, all moments of $e^{-\beta \ell} \varrho(\ell)$ converge (cf. remark following the proof of Proposition 4.2). In particular, the above sum converges. Thus $\delta(\beta)>0$.

Proof of Theorem 4.3. We first note that any surface in the set $\left\{S \in \sigma_{2 L+1} \mid \boldsymbol{r}_{h} \in \operatorname{Int}(S)\right\}$ may be constructed by taking two open tubes in $\varkappa_{L}$ (one "facing" to the right, and the other translated and "facing" to the left) and joining them to a ring of sufficient diameter. If we relax the constraint that the three pieces connect, we may ignore the weight of the plating factor necessary to joint them. Thus

$$
\mathscr{P}_{L}(h ; \beta) \leqq \frac{o_{L}^{2}}{q_{2 L+1}} \sum_{\ell>2 h} \varrho(\ell) e^{-\beta \ell} .
$$

By Eq. (4.14), the sum in (4.28) is bounded above by $e^{-\lambda h}$ for some $\lambda(\beta)>0$ whenever $\beta>\bar{\beta}^{(2)}$. We also have

$$
o_{L}^{2} / q_{2 L+1} \leqq \frac{1}{\delta^{2}(\beta)} q_{L+1}^{2} / q_{2 L+1} \leqq e^{4 \beta} / \delta^{2}(\beta)<\infty,
$$

where the first inequality follows from Lemma 4.4, and the second from subadditivity. Thus

$$
\mathscr{P}_{L}(h ; \beta) \leqq(\text { const }) e^{-\lambda(\beta) h}
$$

for every $L$.

Remark. We have no proof that the tubes undergo a breathing transition. If one does occur, it is possible that the transition is driven by the melting of rings, which would imply $\beta_{b}=\bar{\beta}^{(2)}$. However, as noted in Sect. 2, systems defined as (unnormalized) sums over surfaces often undergo catastrophic transitions at finite temperature. Thus such systems are not guaranteed to survive roughening (or breathing) transitions in the systems they are attempting to model. Indeed, it may be that the melting of rings drives not only a breathing, but also a catastropic destabilization of our system. In the next subsection, we investigate the width of tubes in an ensemble of Bernoulli plaquettes. As remarked prviously, such a system does not undergo a catastrophic transition. Thus, if a breathing transition occurs, the system is guaranteed to exist beyond that point.

(iii) Stability of Constrained Tubes for $p<1 / 2$. Let us turn attention to constrained SOS tubes in the Bernoulli ensemble. The analogue of our criterion for the width of tubes is expressed in terms of the event $f_{L} \subset q_{2 L+1}$ that at least one of the 
SOS tubes contributing to $q_{2 L+1}$ encloses the point $\boldsymbol{r}_{h}$. The quantity of interest is the conditional probability

$$
\mathscr{P}_{L}(h ; p)=\left\langle f_{L} \mid q_{2 L+1}\right\rangle_{p}=\left\langle f_{L}\right\rangle_{p} /\left\langle q_{2 L+1}\right\rangle_{p} .
$$

Our principal result is the analogue of Theorem 4.3. It is shown (Theorem 4.6) that whenever $p<1 / 2, \limsup _{L \rightarrow \infty} \mathscr{P}_{L}(h ; p)$ decays exponentially in $h$. The point $p=1 / 2$ is, of course, the percolation threshold for the two-dimensional (self-dual) bond ensemble.

We remark that there are alternative candidates for an event of the form $f_{L}$. These include: (1) the event that the minimal SOS tube has in its interior the point $\mathbf{r}_{h}$; and (2) the event that $\mathbf{r}_{h}$ is in the connected bond cluster of the origin. Since these events are subsets of the $f_{L}$ defined above, it is clear that our result (Theorem 4.6) implies that the conditional probabilities of the alternative events also decay exponentially in $h$.

The strategy employed is very similar to that of the previous subsection; however, a little care must be taken due to the competition between positive and negative events.

We begin by defining the open tube event

$$
o_{L}^{\prime}=\left\{\omega \in \Omega_{L} \mid \exists S \in u_{L}^{\prime}, S \text { occupied; } \exists(a, b) \in \mathbb{P}_{L},(0,0,0) \in \text { C.C. }((L, a, b))\right\} .
$$

The prime is removed by the placement of an elementary chimney as the origin:

Evidently

$$
o_{L}=\operatorname{ch}(0) \cup o_{L-1}^{\prime} \text {. }
$$

The principal ingredient in our proof of stability is a bound of the opposite type:

Lemma 4.5. Whenever $p<1 / 2$, there exists a constant $A(p)>0$ such that

$$
\left\langle q_{L}\right\rangle_{p} \geqq A(p)\left\langle\sigma_{L}\right\rangle_{p} .
$$

Before proving Lemma 4.5, let us make immediate use of the result.

Theorem 4.6. Whenever $p<1 / 2$, there exists a constant $\theta(p)>0$ such that

$$
\limsup _{L \rightarrow \infty} \mathscr{P}_{L}(h ; p) \leqq(\text { const }) e^{-\theta(p) h} .
$$

Proof. Observe that the bonds in the plane $\mathbb{P}_{L}$ and the (dual) plaquettes in the strip $L-1 / 2<x<L+1 / 2$ form an effective two-dimensional bond-dual bond Bernoulli system. Let $\tilde{\Omega}_{L}$ denote the $\sigma$-algebra of the effective planar system. We may then define $\mathscr{R}_{L}(h) \subset \Omega_{L}$ to be the event that there is a ring of occupied plaquettes in $L$ $-1 / 2<x<L+1 / 2$, surrounding the "origin" (i.e., the $x$-axis) and enclosing the point $\mathbf{r}_{h}$.

It is easily seen that

$$
\mathscr{P}_{L}(h ; p) \leqq\left\langle\sigma_{L}\right\rangle_{p}^{2}\left\langle\mathscr{R}_{L}(h)\right\rangle_{p} /\left\langle q_{2 L+1}\right\rangle_{p} .
$$

Using the bound of Lemma 4.5, together with the obvious inequality

$$
\left\langle q_{L}\right\rangle_{p}^{2} \leqq \frac{1}{p^{4}(1-p)}\left\langle q_{2 L+1}\right\rangle_{p}
$$


we have

$$
\mathscr{P}_{L}(h ; p) \leqq\left\langle\mathscr{R}_{L}(h)\right\rangle_{p} / p^{4}(1-p) A^{2}(p) .
$$

Exponential decay now follows from well-known two-dimensional bond results [24], which give

$$
\left\langle\mathscr{R}_{L}(h)\right\rangle_{p} \leqq(\text { const }) e^{-\theta(p) h}
$$

with $\theta(p)>0$ for $p<1 / 2$.

Proof of Lemma 4.5. For a given configuration in $o_{L-1}$, there are in general many rings of plaquettes in the strip $L-3 / 2<x<L-1 / 2$ which are part of an open SOS tube from the origin. We may partition the event $o_{L-1}$ according to which ring, $\gamma$, is the maximal such ring:

$$
o_{L-1}=\bigcup_{\gamma} o_{L-1}^{\gamma}
$$

It is observed that all in-plane events which may occur inside $\gamma$ are independent of the condition that $\gamma$ be maximal. Should every bond inside $\gamma$ in the plane $\mathbb{P}_{L-1}$ be occupied, then, for any configuration in $o_{L-1}^{\gamma}$, there is a path of occupied bonds connecting the origin to $(L-1,0,0)$ within the tube. If, in addition, the ring $\gamma$ is plated by vertical plaquettes in the plane $\mathbb{P}_{L-1 / 2}$, and if the chimney event $\operatorname{ch}(L)$ occurs, then the configuration is also in $q_{L}$. Since all these events occur in disjoint regions, they are independent. Thus

$$
\left\langle q_{L}\right\rangle_{p} \geqq(\text { const }) \sum_{\gamma}\left\langle o_{L-1}^{\gamma}\right\rangle_{p}\left[p(1-p)^{2}\right]^{\operatorname{Area}(\gamma)},
$$

where $\operatorname{Area}(\gamma)$ denotes the number of plaquettes within the ring $\gamma$. In the above, the constant depends on $p$, but is independent of $L$.

Performing the partial sum over rings of the same length, we obtain

where

$$
\left\langle q_{L}\right\rangle_{p} \geqq(\text { const }) \sum_{k}\left\langle o_{L-1}^{k}\right\rangle_{p} e^{-\alpha k^{2}},
$$

$$
\left\langle o_{L-1}^{k}\right\rangle_{p}=\sum_{\gamma:|\gamma|=k}\left\langle o_{L-1}^{\gamma}\right\rangle_{p}
$$

and $\alpha=$ const $<\infty$. Defining the (normalized) weights $\left.w_{k}=\left\langle\sigma_{L-1}^{k}\right\rangle_{p} / \iota_{L-1}\right\rangle_{p}$, we have

$$
w_{k} \leqq \frac{\left\langle o_{L-2}\right\rangle_{p}}{\left\langle o_{L-1}\right\rangle_{p}}\left\langle v_{k}\right\rangle_{p} \leqq \frac{1}{p^{4}(1-p)}\left\langle v_{k}\right\rangle_{p},
$$

where $v_{k}$ is the event that there is a ring of occupied plaquettes of length $k$ encircling the "origin" in the strip $L-3 / 2<x<L-1 / 2$. Performing a Jensen inequality on (4.43), and using (4.45), we obtain

$$
\left\langle q_{L}\right\rangle \geqq \text { (const) } \exp \left[-\alpha^{\prime} \sum_{k}\left\langle v_{k}\right\rangle_{p} k^{2}\right]\left\langle o_{L-1}\right\rangle_{p}
$$

For $p<1 / 2$, the quantities $\left\langle v_{k}\right\rangle_{p}$ have finite moments of all order. (A simple estimate shows, for example, that $\left\langle v_{k}\right\rangle_{p} \leqq e^{-c \sqrt{k}}$.) Thus the sum in the exponent in (4.46) converges. We may bound $\left\langle o_{L-1}\right\rangle_{p}$ from below by $\left\langle o_{L}\right\rangle_{p}$ to obtain the desired result. 


\section{Entropy of Random Surfaces}

In order to study the entropy of lattice tubes, we rewrite $Q_{L}(\beta)$ in the form (1.3):

$$
Q_{L}(\beta)=\sum_{k} \Gamma_{k}(L) e^{-\beta k L}
$$

Here $\Gamma_{k}(L)$ is the number of surfaces with exactly $k L$ plaquettes, i.e. with "covering factor" $k$. It is easy to see that the necessary and sufficient condition for $\Gamma_{k}(L)>0$ is $k L=2 n \geqq 4 L$, where $n$ is an integer. Thus, for each rational $k \geqq 4$, there is a squence of lengths $\mathscr{L}(k)$ tending to infinity, such that $\Gamma_{k}(L)>0$ for all $L \in \mathscr{L}(k)$. It turns out that the quantities $\Gamma_{k}(L)$ are relatively interesting in their own right. The starting point is to establish that $\Gamma_{k}(L)$ has well-defined asymptotic behavior:

Proposition 5.1. The limit

$$
\zeta(k) \equiv \lim _{L \rightarrow \infty}\left(\frac{1}{L} \log \Gamma_{k}(L) \mid L \in \mathscr{L}(k)\right)
$$

exists and is finite for all rational $k \geqq 4$.

Proof. If $L_{1}, L_{2} \in \mathscr{L}(k)$, then clearly $L_{1}+L_{2} \in \mathscr{L}(k)$. Thus we obtain the subadditive estimate $\Gamma_{k}\left(L_{1}+L_{2}\right) \geqq \Gamma_{k}\left(L_{1}\right) \Gamma_{k}\left(L_{2}\right)$. An elementary argument, with some caution taken to ensure that one does not stray from $L \in \mathscr{L}(k)$, establishes the existence of the limit. The limit $\zeta(k)$ is nonnegative by definition. A Peierls estimate of the form $\Gamma_{k}(L) \leqq e^{c k L}$ shows that $\zeta(k)$ is always finite.

Remarks. (1) As usual, we obtain as a corollary the subadditive bounds

$$
\Gamma_{k}(L) \leqq e^{\zeta(k) L} \quad \forall L .
$$

(2) It is observed that exact subadditivity is not a requirement for the proof of Proposition 5.1 (or for analogous arguments in the rest of this section). In particular, a little patching shows that the $\Gamma$ 's associated with the direct correlation function also have well-defined asymptotic behavior.

The $\zeta(k)$ defined as the limit in (5.2) is of course meaningless for irrational $k$. However, the following proposition demonstrates that $\zeta(k)$ has a natural extension to a function defined on all real numbers.

Proposition 5.2. $\zeta(k)$ is concave as a function of (rational) $k \geqq 4$.

Proof. Let $k_{1}, k_{2} \geqq 4$ be rational. Let $\lambda \in[0,1]$ be rational. Pick $L \in \mathscr{L}\left(\lambda k_{1}\right.$ $\left.+(1-\lambda) k_{2}\right)$ so that $\lambda L \in \mathscr{L}\left(k_{1}\right)$ and $(1-\lambda) L \in \mathscr{L}\left(k_{2}\right)$. By arranging a covering factor of $k_{1}$ in the first $\lambda L$ steps and a covering of $k_{2}$ in the final $(1-\lambda) L$ steps, we obtain

$$
\Gamma_{L}\left(\lambda k_{1}+(1-\lambda) k_{2}\right) \geqq \Gamma_{\lambda L}\left(k_{1}\right) \Gamma_{(1-\lambda) L}\left(k_{2}\right),
$$

from which the desired result can be shown to follow.

Since $\zeta(k)$ is concave, it is also continuous and thus extends to a (concave, continuous) function on all real $k \geqq 4$. A few comments are in order:

(1) Since $\zeta(k) \geqq 0$ for all $k \geqq 4$, the concavity implies that the function is nondecreasing.

(2) Clearly $\zeta(4)=0$. It is sometimes convenient to define $\zeta(k)=-\infty$ for all $k<4$. In this context, it is gratifying to observe that $d \zeta / d k \sim \log (1 /(\mathrm{k}-4))$ as $k \downarrow 4$. 
(i) Thermodynamics of Random Surfaces. The function $\zeta(k)$ has an ad hoc interpretation as the surface entropy at covering factor $k$. Indeed, taking the derivative of $Q_{L}$ with respect to $\beta$, we obtain

$$
-\frac{1}{L} \frac{\partial}{\partial \beta} \log Q_{L}(\beta)=\frac{1}{L} \frac{\sum_{S}|S| e^{-\beta|S|}}{\sum_{S} e^{-\beta|S|}}=\langle k\rangle_{\beta},
$$

i.e., the expected covering at inverse temperature $\beta$. Conversely (after an informal exchange of limits), the left-hand side of (5.5) is seen to "equal" $\partial M / \partial \beta$. This allows the interpretation: $Q \leftrightarrow$ partition function, $M \leftrightarrow$ free energy, $\zeta \leftrightarrow$ entropy, $k \leftrightarrow$ energy, which suggests that there is a canonical relationship between all relevant parameters. That this is indeed the case is shown in the following:

Theorem 5.3. The variables $k$ and $\beta$ are canonically conjugate. Moreover, $M$ and $\zeta$ are related by a Legendre transform, i.e.

$$
-M(\beta)=\sup _{k}[\zeta(k)-\beta k] .
$$

Remark. Since $\zeta(k)$ is concave (Proposition 5.2), we may also express $\zeta(k)$ via the inverse Legendre transform.

Before proceeding with the proof of Theorem 5.3, we must dispense with a minor annoyance. To this end, let $Q_{L}^{T}(\beta) \leqq Q_{L}(\beta)$ represent the correlation function defined by summing only over those surfaces $\mathscr{S}_{L}^{T} \subset \mathscr{S}_{L}$ which are confined to the region $|y|,|z|<T\left(1+L^{2}\right)$. Evidently $M^{T}$ is well-defined, nondecreasing in $T$ and bounded below by $M$.

Lemma 5.4. $\lim _{T \rightarrow \infty} M^{T}=M$.

Proof. The lemma is easily verfied if $M=-\infty$. Now suppose $M>-\infty$. Observe that for any finite $L$,

$$
\lim _{T \rightarrow \infty} Q_{L}^{T}(\beta)=Q_{L}(\beta)
$$

Let $\varepsilon>0$ and choose $L_{0}$ so large that $Q_{L_{0}}(\beta)>\varepsilon^{-(M+\varepsilon) L_{0}}$. By (5.7), for $T$ sufficiently large, $Q_{L_{0}}^{T}(\beta) \geqq Q_{L_{0}}(\beta) e^{-\varepsilon L_{0}}$. Using the subadditive bound on $Q_{L_{0}}^{T}(\beta)$ [i.e., $Q^{T}(\beta)$ $\left.\leqq e^{-M^{T}(\beta)} \forall L\right]$, this implies

$$
M^{T}(\beta) \leqq M(\beta)+2 \varepsilon .
$$

Remark. Although we require only the above result, it will turn out that $M^{T}$ is independent of $T$. That this should be the case is indicated by the observation that, for any fixed $k$ and $T>0, \Gamma_{k}^{T}(L)=\Gamma_{k}(L)$ once $L$ is large enough. Thus $\zeta^{T}(k)=\zeta(k)$ for all $T>0$.

Proof of Theorem 5.3. Expressing $Q_{L}^{T}(\beta)$ in the form (5.1), it is clear that, for any (rational) $k$

$$
Q_{L}^{T}(\beta) \geqq \Gamma_{k}^{T}(L) e^{-\beta k L}=\Gamma_{k}(L) e^{-\beta k L}
$$

for $L$ sufficiently large. This implies

$$
-M^{T}(\beta) \geqq \zeta(k)-\beta k
$$


Taking a supremum over $k$ of (5.10), a lower bound of the form (5.6) is established. If the right-hand side of $(5.10)$ is $+\infty$ [indicating $M^{T}(\beta)=-\infty$ ], we are done. Otherwise, observe that there are only $V_{T}(L) \sim L\left(L^{2}\right)^{d-1}$ nonzero terms in the expression (5.1) for $Q_{L}^{T}(\beta)$. Replacing each nonzero $\Gamma_{k}^{T}(L)$ by $\Gamma_{k}(L)$, we have the upper bound

$$
Q_{L}^{T}(\beta) \leqq \sum_{k}^{V_{T}(L)} \Gamma_{k}(L) e^{-\beta k L} \leqq \sum_{k}^{V_{T}(L)} e^{\zeta(k)-\beta k L} .
$$

Evidently,

$$
\frac{1}{L} \log Q_{L}^{T}(\beta) \leqq \frac{1}{L} \log V_{T}(L)+\sup _{k}[\zeta(k)-\beta k] .
$$

Letting $L \rightarrow \infty$ and using Lemma 5.4, the desired result is established. Note that this also implies $M^{T}=M \forall T$, as expected.

Remark. Standard arguments show that with the possible exception of a single point, $\bar{\beta}$ defined below, whenever $M(\beta)>-\infty$, there is actually a maximizing $k$ for the right-hand side of (5.6). We shall denote this maximizer by $k(\beta)$.

Corollaries. (1) The critical melting temperature, $\beta_{c}$, is given by

$$
\beta_{c}=\sup _{k}[\zeta(k) / k] \text {. }
$$

(2) If

$$
\bar{\beta} \equiv \lim _{k \rightarrow \infty}[\zeta(k) / k]
$$

is strictly positive, then $\bar{\beta}$ is a catastrophic transition point, i.e. $M(\beta)=-\infty$ for $\beta<\bar{\beta}$.

(3) $\forall \beta \geqq \bar{\beta} \quad M(\beta)=M\left(\beta^{+}\right) \equiv \lim _{\varepsilon \downarrow p} M(\beta+\varepsilon)$. In particular, $M(\beta)$ is right continuous at $\bar{\beta}$.

(4) $\forall \beta_{1}, \beta_{2}$ with $\beta_{2} \geqq \beta_{1}, \beta_{1} \geqq \bar{\beta}$,

$$
M\left(\beta_{1}\right)-M\left(\beta_{2}\right) \geqq k_{\min }\left(\beta_{1}-\beta_{2}\right)=4\left(\beta_{1}-\beta_{2}\right) .
$$

(5) If $\beta_{c} \neq \bar{\beta}$, then $M(\beta) \sim\left(\beta-\beta_{c}\right)$ as $\beta \downarrow \beta_{c}$.

Proofs. Corollaries (1) and (2) may be checked by using the identity

$$
-M(\beta)=\sup _{k}\left[\zeta(k)-\beta^{*} k+\left(\beta^{*}-\beta\right) k\right]
$$

with $\beta^{*}=\beta_{c}$ or $\bar{\beta}$, as appropriate.

To prove (3), first assume $M(\bar{\beta})>-\infty$. Let $\delta>0$. We may find a $k_{\delta}$ such that $\zeta\left(k_{\delta}\right)-\bar{\beta} k_{\delta} \geqq-M(\bar{\beta})-\delta$. But then $-M(\bar{\beta}+\varepsilon) \geqq \zeta\left(k_{\delta}\right)-\bar{\beta} k_{\delta}-\varepsilon k_{\delta} \geqq-M(\bar{\beta})-\varepsilon k_{\delta}$ $-\delta$. Letting $\varepsilon \downarrow 0$, the desired result is obtained. A similar analysis shows that if $M(\bar{\beta})=-\infty$, then $M(\beta) \rightarrow-\infty$ as $\beta \downarrow \bar{\beta}$.

To prove (4), first assume $\beta_{1}>\bar{\beta}$. Then, by the remark following the proof of Theorem 5.3, the expression (5.6) for $M\left(\beta_{1}\right)$ has a maximizer $k\left(\beta_{1}\right)$. Evidently

$$
M\left(\beta_{2}\right)-M\left(\beta_{1}\right) \geqq k\left(\beta_{1}\right)\left(\beta_{2}-\beta_{1}\right) .
$$

We note, for future reference, that (5.17) is independent of the relative size of $\beta_{1}$ and $\beta_{2}$. However, for $\beta_{2} \geqq \beta_{1}$, we may replace $k\left(\beta_{1}\right)$ by its lower bound $k_{\min }=4$, the 
minimum covering factor. If $\beta_{1}=\bar{\beta}$, the analysis may be repeated by noting that $\forall \delta>0$, there is a $k_{\delta}$ for which $\zeta(k)-\bar{\beta} k$ is within $\delta$ of its maximum value.

To establish (5), first take $\beta_{1}=\beta_{c} \neq \bar{\beta}$ and $\beta_{2}=\beta \geqq \beta_{c}$ in (5.17). This gives $M(\beta)$ $\geqq 4\left(\beta-\beta_{c}\right)$. Conversely, take $\beta_{2}=\beta_{c} \neq \bar{\beta}$ and $\beta_{1}=\beta \geqq \beta_{c}$ in (5.17). Reversing the inequality, we obtain $M(\beta) \leqq k(\beta)\left(\beta-\beta_{c}\right) \leqq k\left(\beta_{c}\right)\left(\beta-\beta_{c}\right)$. Since $\beta_{c} \neq \bar{\beta}, k\left(\beta_{c}\right)$ is finite.

Remark. We may do a trivial estimate to bound $\Gamma_{k}(L)$ from below by (say) considering rings in a plane which may be serrated at random. Such an estimate shows that $\bar{\beta}>0$, and thus by Corollary (2) that our model has a catastrophic transition.

It is worth noting that the considerations in this section are quite general; indeed, the results may be applied to any model of the form (1.1), provided that the process is (essentially) subadditive. In particular, the results are applicable to random walks as well as random surfaces. For both types of systems, the corollaries to Theorem 5.3 offer simple proofs of rather soft, but often useful properties.

(ii) Analyticity of the Mass and Power Law Corrections to the Entropy. The relationship (5.1) between $Q_{L}(\beta)$ and $\Gamma_{k}(L)$, coupled with the fact that there is a (finite) maximizing $k(\beta)$ (for $\beta>\bar{\beta}$ ), can be used to show that if there is a neighborhood of $k(\beta)$ for which $\Gamma_{k}(L) \sim L^{w} e^{\zeta(k) L}$, then $Q_{L}(\beta) \sim L^{w+1 / 2} e^{-M(\beta) L}$. Unfortunately, direct estimates on the number of surfaces are difficult to obtain. Conversely, it should be possible to use asymptotic estimates on $Q_{L}(\beta)$ to obtain information on the behavior of $\Gamma_{k(\beta)}(L)$. The first step, which is of some independent interest, is to establish analyticity of the mass.

Theorem 5.5. $M(\beta)$ is analytic whenever $M(\beta)<M_{c}(\beta)$.

Proof. By an argument similar to that used in the proof of Lemma 2.6, it can be shown that when $|z|<e^{M_{c}(\beta)}$, the function

$$
\widehat{\mathbb{C}}(z, g) \equiv \sum_{L} z^{L} \sum_{k} \Gamma_{L}^{c}(k) g^{k L}
$$

is regular in $g$ is a neighborhood of $|g|=e^{-\beta}$. Next, recall that whenever $M(\beta)$ $<M_{c}(\beta), e^{M(\beta)}$ may be defined as the smallest real zero of $2-\hat{\mathbb{C}}\left(z, e^{-\beta}\right) / z e^{-4 \beta}$. Analyticity is established by invoking the (analytic) implicit function theorem.

The power law corrections can now be determined by an analysis along the lines of Appendix B.

Theorem 5.6. For all $k$ for which the corresponding $\beta(k)$ satisfies $M(\beta)<M_{c}(\beta)$, the asymptotic behavior of $\Gamma_{k}(L)$ is given by

$$
\Gamma_{k}(L)=(\text { const }) \frac{1}{L^{3 / 2}} e^{\zeta(k) L}\left[1+O\left(\frac{1}{\sqrt{L}}\right)\right] .
$$

Proof. The proof entails asymptotic analysis of the integral

$$
\Gamma_{k}(L)=-\frac{1}{(2 \pi)^{2}} \oint \frac{d g}{g^{k L+1}} \oint \frac{d z}{z^{L+1}} \int_{-\pi}^{+\pi} d \omega_{1} d \omega_{2} \hat{Q}_{L}\left(g, z, \omega_{1}, \omega_{2}\right)
$$


with $\hat{Q}_{L}\left(g, z, \omega_{1}, \omega_{2}\right)$ given by the transformed Ornstein-Zernike equation (2.27), analytically continued to complex values of $g$. Throughout our analysis we will restrict attention to $|g|<e^{-\beta_{0}}$, where $\beta_{0}$ is such that the masses are separated whenever $\beta>\beta_{0}$.

We first observe that if the integration is performed about some fixed circle $g=e^{-\beta} e^{i \varphi}$, then by the $g \leftrightarrow-g$ symmetry of the integrand, we need only integrate the phase of $g$ from $-\pi / 2$ to $\pi / 2$ and double the result. Next, a detailed examination of the expansion of $\mathbb{C}_{3}(g)$ in powers of $g$ shows that the phases are mismatched except at $\varphi=0$. Using this as the key ingredient in an argument along the lines of Lemma B.1, it can be shown that the contribution to (5.20) from the region $|\varphi|>\delta_{\varphi}$ has modulus bounded above by

$$
\text { (const) }\left[e^{-(M(\beta)+\varepsilon) L} e^{+\beta k L}\right]
$$

whenever $\beta>\beta_{0}$. The constant $\varepsilon$ depends, of course, on $\beta$ and $\delta_{\varphi}$. Another argument along the lines of Lemma B.1 establishes that the contribution to (5.20) from $\left(\omega_{1}, \omega_{2}\right)$ outside some small neighborhood of the origin is also bounded above by an estimate of the form (5.21).

Employing a Rouché argument similar to that of Lemma B.2, we may perform the $z$-integration to obtain

$$
\begin{aligned}
\Gamma_{k}(L)= & \oint_{|\varphi|<\delta_{\varphi}} \frac{d g}{g^{k L+1}} \int_{\left|\omega_{1}\right|,\left|\omega_{2}\right|<\delta_{\omega}} d \omega_{1} d \omega_{2} e^{-M\left(g, \omega_{1}, \omega_{2}\right) L} F\left(g, \omega_{1}, \omega_{2}\right) \\
& \cdot\left[1+O\left(e^{-(M(\beta)+\varepsilon) L} e^{\beta k L}\right)\right] .
\end{aligned}
$$

In the above, $\delta_{\varphi}$ and $\delta_{\omega}$ are chosen small enough so that (1) the implicit function $e^{M\left(g, \omega_{1}, \omega_{2}\right)}$ satisfying

$$
0=2-\frac{1}{e^{M\left(g, \omega_{1}, \omega_{2}\right)}} \frac{1}{g^{4}} \hat{\mathbb{C}}\left(e^{M\left(g, \omega_{1}, \omega_{2}\right)}, g, \omega_{1}, \omega_{2}\right)
$$

exists; and (2) the Rouché argument is valid.

Now when $\varphi \neq 0$, both $e^{M(g)}$ and $g^{k}$ develop imaginary parts which, when raised to the $L^{\text {th }}$ power, oscillate so strongly that the purported "error" term dominates the "principal contribution." To avoid this difficulty, we choose $|g| \equiv e^{-\beta}$ so that the integrand has stationary phase. This is done by finding the $\beta$ such that $\partial M(\beta) / \partial \beta=k$, i.e. $\beta=\beta(k)$. By the remark following the proof of Theorem 5.3, $-M(\beta(k))+k \beta(k)$ (which is the coefficient of $L$ in the exponent) is identified as $\zeta(k)$. The $\omega_{1}, \omega_{2}$ and $\varphi$ integrations are now easily performed; each multiplies the result by a factor of $L^{-1 / 2}$.

\section{Appendix A}

\section{Regularity of the Transform Functions for Percolation}

Here we provide a proof of Lemma 3.6 for the function $\hat{Q}_{p}\left(z, \xi_{1}, \xi_{2}\right)$; the proof for $\hat{C}_{p}\left(z, \xi_{1}, \xi_{2}\right)$ is identical.

For each pair $\left(k_{a}, k_{b}\right)$ of rational numbers, we define the correlation function

$$
Q_{L}^{\left(k_{a}, k_{b}\right)}=\left\langle\mathscr{Q}_{L,\left(k_{a} L, k_{b} L\right)}\right\rangle_{p} .
$$

This function is to be understood in the sense of Sect. 5; that is, we consider only those $L \in \mathscr{L}\left(k_{a}, k_{b}\right)$ for which $Q_{L}^{\left(k_{a}, k_{b}\right)}$ need not vanish. 
Proposition A.1. Let $k_{a}$ and $k_{b}$ be rational. Then $\forall p \in(0,1)$

$$
\lim _{L \rightarrow \infty}\left(-\frac{1}{L} \log Q_{L}^{\left(k_{a}, k_{b}\right)} \mid L \in \mathscr{L}\left(k_{a}, k_{b}\right)\right) \equiv \mathscr{M}\left(k_{a}, k_{b}\right)
$$

exists in $\mathbb{R}^{*}$. Furthermore $\mathscr{M}\left(k_{a}, k_{b}\right)$ is jointly convex in $k_{a}$ and $k_{b}$.

Proof. The existence of the mass follows from the usual subadditive bound

$$
Q_{L_{1}+L_{2}}^{\left(k_{a}, k_{b}\right)} \geqq f(p) Q_{L_{1}}^{\left(k_{a}, k_{b}\right)} Q_{L_{2}}^{\left(k_{a}, k_{b}\right)}
$$

where $f(p)>0$ is the patching factor required to join the tubes. Now simply iterate (A.3) and take $\lim _{L \rightarrow \infty} \frac{1}{L} \log (\cdot)$ through a sequence $\left(L \in \mathscr{L}\left(k_{a}, k_{b}\right)\right)$.

Convexity in $k_{a}$ is a consequence of the subadditive estimate

$$
Q_{L}^{\left(\lambda k_{a}+(1-\lambda) k_{a}^{\prime}, k_{b}\right)} \geqq f(p) Q_{\lambda L}^{\left(k_{a}, k_{b}\right)} Q_{(1-\lambda) L}^{\left(k_{a}^{\prime}, k_{b}\right)},
$$

for rational $\lambda \in[0,1]$. Again the limit must be taken through a sequence $(L)$ such that $L \in \mathscr{L}\left(\lambda k_{a}+(1-\lambda) k_{a}^{\prime}, k_{b}\right), \lambda L \in \mathscr{L}\left(k_{a}, k_{b}\right)$ and $(1-\lambda) L \in \mathscr{L}\left(k_{a}^{\prime}, k_{b}\right)$.

Remark. The usual subadditive bounds (cf. corollary to Proposition 2.1) imply

$$
Q_{L}^{\left(k_{a}, k_{b}\right)} \leqq e^{-\mathscr{M}\left(k_{a}, k_{b}\right) L} \quad \forall L .
$$

Furthermore, by convexity, we may extend $\mathscr{M}\left(k_{a}, k_{b}\right)$ to a continuous function of its arguments.

Since $\mathscr{M}\left(k_{a}, k_{b}\right)$ is convex and symmetric in $k_{a} \leftrightarrow-k_{a}, k_{b} \leftrightarrow-k_{b}$, it must be either a constant or, eventually, increasing (at least linearly) with $\left|k_{a}\right|,\left|k_{b}\right|$. In the latter case, the contribution to the correlation function from surfaces outside some fixed cone is negligible. The following proposition, which makes use of the asymptotic decay of the connectivity function, shows that, indeed, the latter case is realized. This enables us to prove the desired regularity.

Proposition A.2. Whenever $p<\sigma_{c}$, there exist constants $\lambda_{1}, \lambda_{2}>0$ such that $\mathscr{M}\left(k_{a}, k_{b}\right)$ $\geqq \lambda_{1}+\lambda_{2}\left(\left|k_{a}\right|+\left|k_{b}\right|\right)$.

Proof. This follows from the obvious fact that $\forall k_{a}, k_{b}$ rational, and for $L$ sufficiently large,

$$
Q_{L}^{\left(k_{a}, k_{b}\right)} \leqq \Lambda_{E_{0}, E_{\left(L, k_{a} L, k_{b} L\right)}} \leqq e^{-\lambda\left(1+\left|k_{a}\right|+\left|k_{b}\right|\right) L} .
$$

(The second inequality is simply Proposition 3.5.)

Proof of Lemma 3.6. It suffices to show that for $|z|<e^{M(p)}$, and for $\left|\xi_{1}\right|,\left|\xi_{2}\right|$ in some neighborhood of 1 , the sum

$$
\hat{Q}_{p}\left(z, \xi_{1}, \xi_{2}\right)=\sum_{L, a, b}\left\langle\mathscr{Q}_{L,(a, b)}\right\rangle_{p} z^{L} \xi_{1}^{a} \xi_{2}^{b}
$$

is absolutely convergent.

First note that $M(p)=\mathscr{M}(0,0)$. By the above proposition, there exists $\bar{k}_{a}, \bar{k}_{b}$ and $\lambda>0$ such that

$$
\mathscr{M}\left(k_{a}, k_{b}\right) \geqq M(p)+\lambda\left[\left(k_{a}-\bar{k}_{a}\right)+\left(k_{b}-\bar{k}_{b}\right)\right] .
$$

Take $\left|\xi_{1}\right|$ and $\left|\xi_{2}\right|$ to lie within a disk of radius $e^{\lambda}$. 
Since $|z|<e^{M(p)}$, there exists a neighborhood of $\left|\xi_{i}\right|=1$ (possibly smaller than $e^{\lambda}$ ) such that for $\left|\xi_{1}\right|$ and $\left|\xi_{2}\right|$ in this neighborhood,

$$
|z|\left|\xi_{1}\right|^{\bar{k}_{a}}\left|\xi_{2}\right|^{\bar{k}_{b}}<e^{M(p)} .
$$

We take $\left|\xi_{1}\right|$ and $\left|\xi_{2}\right|$ to lie inside the smaller of the two regions specified above.

Now using the subadditive bound on the master function

and the bound (A.5)

$$
\mathbb{Q}_{L}(p) \equiv \sum_{a, b}\left\langle\mathscr{Q}_{L,(a, b)}\right\rangle_{p} \leqq e^{-M(p) L},
$$

$$
\left\langle\mathscr{Q}_{L,(a, b)}\right\rangle_{p} \leqq e^{-\mathscr{M}(a / L, b / L) L},
$$

it is straightforward to verify absolute convergence of the (triple) sum (A.7).

\section{Appendix B}

Asymptotic Behavior of $Q_{L,(a, b)}$

In this appendix, we analyze the integral

$$
Q_{L,(a, b)}=\frac{1}{2 \pi i} \oint \frac{d z}{z^{L+1}} \int_{-\pi}^{\pi} d \omega_{1} d \omega_{2} e^{-i a \omega_{1}} e^{-i b \omega_{2}} \frac{z g^{4}}{2-\frac{1}{z g^{4}} \hat{C}\left(z,\left(\omega_{1}, \omega_{2}\right)\right)} .
$$

For $a=b=0$, this analysis completes the proofs of Theorems 2.7 and 3.7. The general case is needed for the proof of Theorem 4.1.

We first demonstrate that as $L \rightarrow \infty$, the only significant contribution to the integral is from an infinitesimal neighborhood of $\omega_{1}=\omega_{2}=0$.

Lemma B.1. $\forall \delta>0, \exists \varepsilon, v>0$ such that unless $\left|\omega_{1}\right|,\left|\omega_{2}\right|<\delta$

$\forall z$ with $|z| \leqq e^{M+\varepsilon}$.

$$
\left|2-\frac{1}{z g^{4}} \hat{C}\left(z,\left(\omega_{1}, \omega_{2}\right)\right)\right|>\frac{v|z|^{2}}{g^{4}}
$$

Using the bound (B.2), we may integrate (B.1) around the circle $|z|=e^{M+\varepsilon}$ to obtain:

\section{Corollary.}

$$
\begin{aligned}
& \left|\frac{1}{2 \pi i} \oint \frac{d z}{z^{L+1}} \int_{\left|\omega_{1}\right|>\delta} d \omega_{1} \int_{\left|\omega_{2}\right|>\delta} d \omega_{2} \frac{z g^{4}}{2-\frac{1}{z g^{4}} \hat{C}\left(z,\left(\omega_{1}, \omega_{2}\right)\right)}\right| \\
& \quad \leqq \frac{(2 \pi)^{2}}{v} g^{8} e^{-(M+\varepsilon)(L+1)} .
\end{aligned}
$$

Note that the upper bound in (B.3) is exponentially small relative to the (anticipated) value of the integral (B.1).

Proof of Lemma B.1. It is convenient to express $\hat{C}\left(z,\left(\omega_{1}, \omega_{2}\right)\right)$ as a power series in $z$ with coefficients $C_{L}\left(\omega_{1}, \omega_{2}\right)$. By the $a \leftrightarrow-a, b \leftrightarrow-b$ symmetry, each $C_{L}\left(\omega_{1}, \omega_{2}\right)$ is of the form of a cosine series with positive coefficients, and hence is maximized by 
$\omega_{1}=\omega_{2}=0$. Furthermore, the maximum is quadratic whenever $C_{L}\left(\omega_{1}, \omega_{2}\right)$ has nontrivial $\omega$-dependence. This turns out to be the case for all $L \geqq 3$. Thus, given $\delta>0$, there exists $v>0$ such that

$$
\sup _{\substack{\left|\omega_{1}\right|>\delta \\\left|\omega_{2}\right|>\delta}}\left|C_{3}\left(\omega_{1}, \omega_{2}\right)\right|<C_{3}(0,0)-2 v .
$$

It follows that, for $\left|\omega_{1}\right|,\left|\omega_{2}\right|>\delta$

$$
\begin{aligned}
\left|\hat{C}\left(z,\left(\omega_{1}, \omega_{2}\right)\right)\right| & \leqq \mathbb{C}_{1}|z|+\mathbb{C}_{2}|z|^{2}+\left|C_{3}\left(\omega_{1}, \omega_{2}\right)\right||z|^{3}+\mathbb{C}_{4}|z|^{4}+\ldots \\
& =\hat{\mathbb{C}}(|z|)+|z|^{3}\left[C_{3}\left(\omega_{1}, \omega_{2}\right)-C_{3}(0,0)\right] \leqq \widehat{C}(|z|)-2 v|z|^{3} .
\end{aligned}
$$

Next, we find an $\varepsilon>0$ small enough so that

$$
\left.\frac{\widehat{C}(x)}{x g^{4}}\right|_{x=e^{M+\varepsilon}}<2+v e^{2 M} / g^{4} .
$$

[Recall that $\widehat{\mathbb{C}}\left(e^{M}\right) / e^{M} g^{4}=2$ and that $[\hat{\mathbb{C}}(x) / x]^{\prime}>0$ for $x$ real and positive.] Since the coefficients, $\mathbb{C}_{L}$, are nonnegative, (B.6) implies

$$
\left|\widehat{\mathbb{C}}(z) / z g^{4}\right| \leqq \widehat{\mathbb{C}}(|z|) /|z| g^{4}<2+v e^{2 M} / g^{4},
$$

whenever $e^{M} \leqq|z| \leqq e^{M+\varepsilon}$. When $|z|<e^{M}$, we have the (stronger) bound

$$
\left|\widehat{\mathbb{C}}(z) / z g^{4}\right| \leqq \widehat{\mathbb{C}}(|z|) /|z| g^{4}<2 .
$$

Combining (B.5), (B.7), and (B.8), we have

$$
\begin{aligned}
\left|2-\frac{1}{z g^{4}} \hat{\mathbb{C}}\left(z,\left(\omega_{1}, \omega_{2}\right)\right)\right| & \geqq 2-\hat{\mathbb{C}}(|z|) /|z| g^{4}+2 v|z|^{2} / g^{4} \\
& \geqq 2 v|z|^{2} / g^{4}-\chi\left(|z| \geqq e^{M}\right) v e^{2 M} / g^{4} \\
& \geqq v|z|^{2} / g^{4} .
\end{aligned}
$$

In the above, $\chi\left(|z| \geqq e^{M}\right)=1$ if $|z| \geqq e^{M}$, and zero otherwise.

By the reasoning in the proof of Theorem 2.7, the function $2-\hat{C}(z,(0,0)) / z g^{4}$ has a simple zero at $z=e^{M}$, and no other zeroes in the annulus $e^{M}<|z| \leqq e^{M} \mid \lambda$. We now verify that a similar situation occurs for $\left(\omega_{1}, \omega_{2}\right)$ sufficiently close to $(0,0)$.

Lemma B.2. For any $A>0$ satisfying $e^{M}+A<\lambda^{-1} e^{M}, \exists \delta>0$ such that for all $\left|\omega_{1}\right|$, $\left|\omega_{2}\right|<\delta,\left[2-\hat{C}\left(z,\left(\omega_{1}, \omega_{2}\right)\right) / z g^{4}\right]$ has exactly one zero inside the disk $|z|<e^{M}+A$.

Proof. Define $\kappa=\min _{|z|=e^{M}+A}\left|2-\widehat{\mathbb{C}}(z) / z g^{4}\right|>0$. By choosing $L_{0}$ and $k_{0}$ sufficiently large, we may ensure that

$$
\sum_{L>L_{0}} \mathbb{C}_{L}\left(e^{M}+A\right)^{L}+\sum_{\substack{L<L_{0} \\|a|,|b|>k_{0}}} C_{L,(a, b)}\left(e^{M}+A\right)^{L}<\frac{\kappa}{3}\left(e^{M}+A\right) g^{4} .
$$


An analogous estimate holds for $\omega_{1}$ and $\omega_{2}$ nonzero. Thus, on the circle $|z|=e^{M}+A$, we have

$$
\begin{aligned}
& \left|\frac{1}{z g^{4}} \hat{\mathbb{C}}(z)-\frac{1}{z g^{4}} \hat{C}\left(z,\left(\omega_{1}, \omega_{2}\right)\right)\right|<\frac{2 \kappa}{3} \\
& \quad+\left|\frac{1}{z g^{4}} \sum_{\substack{L<L_{0} \\
|a|,|b|<k_{0}}} C_{L,(a, b)} z^{L}\left(1-e^{i \omega_{1} a} e^{i \omega_{2} b}\right)\right| .
\end{aligned}
$$

Since the second term on the right-hand side of (B.11) is a finite sum, we may find a $\delta>0$ so that it is bounded above by $\kappa / 3$ whenever $\left|\omega_{1}\right|,\left|\omega_{2}\right|<\delta$. Applying the theorem of Rouché, the desired result follows.

To complete the arguments of Theorems 2.7, 3.7, and 4.1, one finds a $\delta^{\prime}$ neighborhood of the origin for which the implicit function theorem defines a function $e^{M\left(\omega_{1}, \omega_{2}\right)}$ describing the motion of the zero specified in Lemma B.2. That $e^{M\left(\omega_{1}, \omega_{2}\right)}$ has a quadratic minimum follows from the form of the coefficients $C_{L}\left(\omega_{1}, \omega_{2}\right)$.

Remark. Regularity of $\hat{C}$ in $\left(\omega_{1}, \omega_{2}\right)$ has been established in Lemma 2.6 and Appendix A. It should be noted that the implicit function theorem (and thus Theorems 2.7 and 3.7) requires only differentiability of $\hat{C}$. However, regularity is necessary for the distortion of the contour (i.e., completing the square) in the proof of Theorem 4.1 .

Acknowledgements. Two of us (J.T.C and L.C.) would like to thank Jürg Fröhlich for several enlightening discussions on free random surface models. We would also like to thank Arthur Jaffe for useful discussions on various aspects of the work.

\section{References}

1. Fröhlich, J.: Quantum field theory in terms of random walks and random surfaces (Cargèse, 1983)

2. Abraham, D.B., Chayes, J.T., Chayes, L.: Statistical mechanics of lattice tubes. Phys. Rev. D 30, 841 (1984)

3. Abraham, D.B.: Two-point functions and bubbles. Phys. Rev. Lett. 50, 291 (1983)

4. Aizenman, M., Chayes, J.T., Chayes, L., Fröhlich, J., Russo, L.: On a sharp transition from area law to perimeter law in a system of random surfaces. Commun. Math. Phys. 92,19 (1983)

5. Durhuus, B., Fröhlich, J., Jónsson, T.: Self-avoiding and planar random surfaces on the lattice. Nucl. Phys. B 225 [FS9], 185 (1983)

6. Durhuus, B., Fröhlich, J., Jónsson, T.: Critical properties of a model of planar random surfaces. Phys. Lett. B (to appear)

7. Ornstein, L.S., Zernike, F.: Proc. Acad. Sci., Amst. 17, 793 (1914)

8. Abraham, D.B., Kunz, H.: Ornstein-Zernike theory of classical fluids at low density. Phys. Rev. Lett. 39, 1011 (1977)

9. Fisher, M.E.: Correlation functions and the critical region of simple fluids. J. Math. Phys. 5, 944 (1964)

10. Brydges, D., Spencer, T.: Self-avoiding walk in 5 or more dimensions. Commun. Math. Phys. (to appear)

11. Harris, T.E.: A lower bound for the critical probability in a certain percolation process. P. Camb. Phil. Soc. 56, 13 (1960) 
12. Fortuin, C., Kasteleyn, P., Ginibre, J.: Correlation inequalities on some partially ordered sets. Commun. Math. Phys. 22, 89 (1971)

13. Kesten, H.: Percolation theory for mathematicians. Boston: Birkhäuser 1982

14. Chayes, J.T., Chayes, L.: The correct extension of the Fortuin-Kasteleyn result to plaquette percolation. Nucl. Phys. B 235 [FS 11], 19 (1984)

15. Kesten, H.: Analyticity properties and power law estimates of functions in percolation theory. J. Stat. Phys. 25, 717 (1981)

16. Lieb, E.H.: A refinement of Simon's correlation inquality. Commun. Math. Phys. 77, 127 (1980)

17. Simon, B.: Correlation inequalities and the decay of correlations in ferromagnets. Commun. Math. Phys. 77, 111 (1980)

18. Aizenman, M., Newman, C.: J. Stat. Phys. (to appear)

19. Chayes, L.: Thesis (Princeton, 1983)

20. Lüscher, M., Symanzik, K., Weisz, P.: Anomalies of the free loop wave equation in the WKB approximation. Nucl. Phys. B 173, 365 (1980)

21. Wu, T.T.: Theory of Toeplitz determinants and the spin correlations of the two-dimensional Ising model. I. Phys. Rev. 149, 380 (1966)

22. Abraham, D.B.: Solvable model with a roughening transition for a planar Ising ferromagnet. Phys. Rev. Lett. 44, 1165 (1980)

23. van Beijeren, H.: Interface sharpness in the Ising system. Commun. Math. Phys. 40, 1 (1975)

24. Kesten, H.: On the time constant and path length of first passage percolation. Adv. Appl. Prob. 12, 848 (1980)

Communicated by A. Jaffe

Received May 3, 1984; in revised form July 30, 1984

Note added in proof: It has recently been established that $M_{c}(\beta)>M(\beta)$ for all $\beta>\bar{\beta}$. Thus Theorems 2.7 and 4.1 give the asymptotic behavior of $Q_{L,(a, b)}(\beta)$ for all noncritical temperatures. It has also been shown that the surfaces contributing to the pair correlation $Q_{L(a, b)}(\beta)$ do not breathe whenever $M_{c}(\beta)>M(\beta)$, and thus for all $\beta>\bar{\beta}$. These results appear in "Nonperturbative analysis of a model of random surfaces," Harvard University preprint (HUTMP 169), by the authors. 
